\title{
Constant Sign and Nodal Solutions for Problems with the $p$-Laplacian and a Nonsmooth Potential Using Variational Techniques
}

\author{
Ravi P. Agarwal, ${ }^{1}$ Michael E. Filippakis, ${ }^{2}$ Donal O' $^{\prime}$ egan, ${ }^{3}$ \\ and Nikolaos S. Papageorgiou ${ }^{4}$ \\ ${ }^{1}$ Department of Mathematical Sciences, Florida Institute of Technology, Melbourne, FL 32901-6975, USA \\ ${ }^{2}$ Department of Mathematics, Hellenic Army Academy, Vari, 16673 Athens, Greece \\ ${ }^{3}$ Department of Mathematics, National University of Ireland, Galway, Ireland \\ ${ }^{4}$ Department of Mathematics, National Technical University, Zografou Campus, 15780 Athens, Greece
}

Correspondence should be addressed to Ravi P. Agarwal, agarwal@fit.edu

Received 10 December 2008; Revised 21 January 2009; Accepted 23 January 2009

Recommended by Juan J. Nieto

\begin{abstract}
We consider a nonlinear elliptic equation driven by the $p$-Laplacian with a nonsmooth potential (hemivariational inequality) and Dirichlet boundary condition. Using a variational approach based on nonsmooth critical point theory together with the method of upper and lower solutions, we prove the existence of at least three nontrivial smooth solutions: one positive, the second negative, and the third sign changing (nodal solution). Our hypotheses on the nonsmooth potential incorporate in our framework of analysis the so-called asymptotically $p$-linear problems.

Copyright (C) 2009 Ravi P. Agarwal et al. This is an open access article distributed under the Creative Commons Attribution License, which permits unrestricted use, distribution, and reproduction in any medium, provided the original work is properly cited.
\end{abstract}

\section{Introduction}

The aim of this work is to prove the existence of multiple solutions of constant sign and of nodal solutions (sign changing solutions) for nonlinear elliptic equations driven by the $p$ Laplacian and having a nonsmooth potential (hemivariational inequalities). So let $Z \subseteq \mathbb{R}^{\mathbb{N}}$ be a bounded domain with a $C^{2}$-boundary $\partial Z$. The problem under consideration is the following:

$$
\begin{gathered}
-\operatorname{div}\left(\|D x(z)\|^{p-2} D x(z)\right) \in \partial j(z, x(z)) \quad \text { a.e. on } Z, \\
\left.x\right|_{\partial Z}=0 \quad 1<p<\infty .
\end{gathered}
$$

Here $j(z, x)$ is measurable function on $Z \times \mathbb{R}$, which in the $x \in \mathbb{R}$ variable is locally Lipschitz and $\partial j(z, x)$ stands for the generalized subdifferential of $x \rightarrow j(z, x)$ in the sense of 
Clarke [1]. Problem (1.1) is a hemivariational inequality. Hemivariational inequalities are a new type of variational expressions, which arise in applications if one considers more realistic mechanical laws of multivalued and nonmonotone nature. Then the corresponding energy (Euler) functional is nonsmooth and nonconvex. Various engineering applications of hemivariational inequalities can be found in the book of Naniewicz-Panagiotopoulos [2].

Multiple solutions of constant sign for problems monitored by the $p$-Laplacian and with a $C^{1}$-potential were obtained by Ambrosetti et al. [3], García Azorero-Peral Alonso [4], and García Azorero et al. [5]. In all these works, the authors consider nonlinear eigenvalue problems and prove the existence of positive and negative solutions for certain values of the parameter $\lambda \in \mathbb{R}$. The question of existence of nodal solutions was first addressed within the framework of semilinear problems (i.e., $p=2$ ). We mention the works of Dancer-Du [6] and Zhang-Li [7], which contain two different approaches to the problem. In Dancer-Du [6], the authors use a combination of the variational method (critical point theory) with the method of upper and lower solutions. In contrast Zhang-Li [7] use invariance properties of the negative gradient flow of the corresponding equation in $C_{0}^{1}(\bar{Z})$. Recently these methods were extended to "smooth" problems driven by the $p$-Laplacian differential operator. CarlPerera [8] extended the work of Dancer-Du [6], by assuming the existence of upper and lower solutions for the problem. Zhang-Li [9] and Zhang et al. [10] extended the semilinear work of [7], by carefully constructing a pseudogradient vector field whose descent flow exhibits the necessary invariance properties. These works were extended recently by FilippakisPapageorgiou [11]. Recently the approach based on the invariance properties of descent flow was used by Zhang-Perera [12] to produce nodal solutions for certain Kirchhoff type equations. Other recent works dealing with $p$-Laplacian equations are those by Ahmad-Nieto [13] (monotone iterative technique), Kim et al. [14] (radial solutions), Lin et al. (singular odes) [15], and Väth [16] (degree theoretic approach).

In this paper using techniques from nonsmooth critical point theory in conjunction with the method of upper and lower solutions, we are able to extend the works of Dancer$\mathrm{Du}$ [6] and Carl-Perera [8] to hemivariational inequalities. Helpful in this respect is the nonsmooth second deformation lemma of Corvellec [17]. Recently, sign-changing solutions for problems with discontinuous nonlinearities were obtained by Averna et al. [18], but in contrast to our work they deal with $p$-superlinear problems.

\section{Mathematical Background}

In our analysis of problem (1.1), we use the nonsmooth critical point theory which is based on the subdifferential theory for locally Lipschitz functions and some basic facts about the spectrum of the negative $p$-Laplacian with Dirichlet boundary conditions. For easy reference, we recall some definitions and results from these areas, which will be used in the sequel.

We start with the subdifferential theory for locally Lipschitz functions and the corresponding nonsmooth critical point theory. Details can be found in the books of Gasiński -Papageorgiou [19] and Motreanu-Panagiotopoulos [20]. So let $X$ be a Banach space and let $X^{*}$ be its topological dual. By $\left.\langle\cdot, \cdot\rangle\right\rangle$ we denote the duality brackets for the pair $\left(X, X^{*}\right)$. Given a locally Lipschitz function $\varphi: X \rightarrow \mathbb{R}$, the generalized directional derivative $\varphi^{0}(x ; h)$ of $\varphi$ at $x \in X$ in the direction $h \in X$ is defined as follows:

$$
\varphi^{0}(x ; h)=\limsup _{\substack{x^{\prime} \rightarrow x \\ \lambda \downarrow 0}} \frac{\varphi\left(x^{\prime}+\lambda h\right)-\varphi\left(x^{\prime}\right)}{\lambda} .
$$


The function $h \rightarrow \varphi^{0}(x ; h)$ is sublinear continuous and so it is the support function of a nonempty, convex, and $w^{*}$-compact set $\partial \varphi(x) \subseteq X^{*}$ defined by

$$
\partial \varphi(x)=\left\{x^{*} \in X^{*}:\left\langle x^{*}, h\right\rangle \leq \varphi^{0}(x ; h) \forall h \in X\right\} .
$$

The multifunction $x \rightarrow \partial \varphi(x)$ is called the "generalized gradient" (or generalized subdifferential) of $\varphi$. If $\varphi: X \rightarrow \mathbb{R}$ is also convex, then $\partial \varphi(x)$ coincides with the subdifferential in the sense of convex analysis $\partial_{c} \varphi(x)$, defined by

$$
\partial_{c} \varphi(x)=\left\{x^{*} \in X^{*}:\left\langle x^{*}, y-x\right\rangle \leq \varphi(y)-\varphi(x) \forall y \in X\right\} .
$$

Moreover if $\varphi \in C^{1}(X)$, then $\varphi$ is locally Lipschitz and $\partial \varphi(x)=\left\{\varphi^{\prime}(x)\right\}$.

We say that $x \in X$ is a critical point of the locally Lipschitz function $\varphi: X \rightarrow \mathbb{R}$, if $0 \in \partial \varphi(x)$. It is easy to see that if $x \in X$ is a local extremum of $\varphi$ (i.e., a local minimum or a local maximum of $\varphi$ ), then $x \in X$ is a critical point of $\varphi$.

A locally Lipschitz function $\varphi: X \rightarrow \mathbb{R}$ satisfies the Palais-Smale condition at level $c \in \mathbb{R}\left(P S_{c}\right.$-condition for short), if every sequence $\left\{x_{n}\right\}_{n \geq 1} \subseteq X$ such that $\varphi\left(x_{n}\right) \rightarrow c$ and $m\left(x_{n}\right)=\inf \left\{\left\|x^{*}\right\|: x^{*} \in \partial \varphi\left(x_{n}\right)\right\} \rightarrow 0$ as $n \rightarrow \infty$ has a strongly convergent subsequence. We say that $\varphi$ satisfies the $P S$-condition, if it satisfies the $P S_{c}$-condition for every $c \in \mathbb{R}$.

The following topological notion is crucial in the minimax characterization of the critical values of a locally Lipschitz functional $\varphi: X \rightarrow \mathbb{R}$.

Definition 2.1. Let $Y$ be a Hausdorff topological space and $E_{0}, E$, and $D$ are nonempty closed subsets of $Y$ with $E_{0} \subseteq E$. We say that the pair $\left\{E_{0}, E\right\}$ is linking with $D$ in $Y$ if and only if

(a) $E_{0} \cap D=\varnothing$;

(b) for any $\gamma \in C(E, Y)$ such that $\left.\gamma\right|_{E_{0}}=\left.i d\right|_{E_{0}}$, we have $\gamma(E) \cap D \neq \varnothing$.

Using this notion, we have the following general minimax principle for the critical values of a locally Lipschitz function $\varphi: X \rightarrow \mathbb{R}$.

Theorem 2.2. If $X$ is a reflexive Banach space, $E_{0}, E$, and $D$ are nonempty closed subsets of $X$ such that $\left\{E_{0}, E\right\}$ is linking with $D$ in $X, \varphi: X \rightarrow \mathbb{R}$ is locally Lipschitz, $\sup _{E_{0}} \varphi<\inf _{D} \varphi, \Gamma=$ $\left\{\gamma \in C(E, X):\left.\gamma\right|_{E_{0}}=\left.i d\right|_{E_{0}}\right\}, c=\inf _{\gamma \in \Gamma} \sup _{v \in E} \varphi(\gamma(v))$, and $\varphi$ satisfies the PS $S_{c}$-condition, then $c \geq \inf _{D} \varphi$ and $c$ is a critical value of $\varphi$.

Remark 2.3. From this general minimax principle, by appropriate choices of the linking sets, one can produce nonsmooth versions of the mountain pass theorem, of the saddle point theorem, and of the generalized mountain pass theorem.

Definition 2.4. If $Y$ is a subset of the Banach space $X$, a "deformation of $Y$ " is a continuous map $h:[0,1] \times Y \rightarrow Y$ such that $h(0, \cdot)=i d_{Y}$. If $V \subseteq Y$, then we can say that $V$ is a "weak deformation retract of $Y^{\prime \prime}$, if there exists a deformation $h:[0,1] \times Y \rightarrow Y$ such that $h(1, Y) \subseteq V$ and $h(t, \cdot) \subseteq V$ for all $t \in[0,1]$. 
Given a locally Lipschitz function $\varphi: X \rightarrow \mathbb{R}$ and $c \in \mathbb{R}$, we define

$$
\begin{aligned}
\stackrel{0}{\varphi^{c}} & =\{x \in X: \varphi<c\}, \\
K_{c} & =\{x \in x: 0 \in \partial \varphi(x), \varphi(x)=c\} .
\end{aligned}
$$

The next theorem is a partial extension to a nonsmooth setting of the so-called "second deformation theorem" (see, e.g., Gasiński -Papageorgiou [21, page 628]) and it is due to Corvellec [17]. In fact the result of Corvellec is formulated in the more general context of metric spaces, for continuous functions using the so-called weak slope. For our purposes, it suffices to use a particular form of the result which we state next.

Theorem 2.5. If $X$ is a Banach space, $\varphi: X \rightarrow \mathbb{R}$ is locally Lipschitz and satisfies the PS-condition, $a \in \mathbb{R}, b \in \mathbb{R} \cup\{+\infty\}, \varphi$ has no critical points in $\varphi^{-1}(a, b)$, and $K_{a}$ is discrete nonempty, then there exists a deformation $h:[0,1] \times \varphi^{b} \rightarrow \varphi^{b}$ such that

(a) $\left.h(t, \cdot)\right|_{K_{a}}=i d$ for all $t \in[0,1]$;

(b) $h\left(1, \varphi^{b}\right) \subseteq \varphi^{a} \cup K_{a}$;

(c) $\varphi(h(t, x)) \leq \varphi(x)$ for all $t \in[0,1]$ and all $x \in \varphi^{b}$.

In particular the set $\varphi^{a} \cup K_{a}$ is a weak deformation retract of $\varphi^{b}$.

Next let us recall some basic facts about the spectrum of the negative $p$-Laplacian with Dirichlet boundary conditions. So let $Z \subseteq \mathbb{R}^{\mathbb{N}}$ be a bounded domain with a $C^{2}$-boundary $\partial Z$ and $m \in L^{\infty}(Z)_{+}, m \neq 0$. We consider the following nonlinear weighted (with weight $m$ ) eigenvalue problem:

$$
\begin{gathered}
-\operatorname{div}\left(\|D x(z)\|^{p-2} D x(z)\right)=\hat{\lambda} m(z)|x(z)|^{p-2} x(z) \quad \text { a.e. on } Z, \\
\left.x\right|_{\partial Z}=0 \quad 1<p<\infty .
\end{gathered}
$$

The least number $\hat{\imath} \in \mathbb{R}$ for which problem (2.5) has a nontrivial solution is the first eigenvalue of $\left(-\Delta_{p}, W_{0}^{1, p}(Z), m\right)$ and it is denoted by $\hat{\lambda}_{1}(m)$. The first eigenvalue $\hat{\lambda}_{1}(m)$ is strictly positive (i.e., $\hat{\lambda}_{1}(m)>0$ ); it is isolated and it is simple (i.e., the associated eigenspace is one dimensional). Moreover, using the Rayleigh quotient we have a variational characterization of $\hat{\mathcal{\lambda}}_{1}(m)$, namely,

$$
\widehat{\lambda}_{1}(m)=\min \left[\frac{\|D x\|_{p}^{p}}{\int_{Z} m|x|^{p} d z}: x \in W_{0}^{1, p}(Z), x \neq 0\right]
$$

(see also Cuccu et al. [22]).

The minimum in (2.6) is attained on the corresponding one-dimensional eigenspace. In what follows by $u_{1} \in W_{0}^{1, p}(Z)$ we denote the normalized eigenfunction. Note that $\left|u_{1}\right|$ also realizes the minimum in (2.6). Hence we may assume that $u_{1}(z) \geq 0$ a.e. on $Z$. Moreover, from nonlinear regularity theory (see, e.g., Gasiński -Papageorgiou [21, page 738]), we have 
$u_{1} \in C_{0}^{1}(\bar{Z})=\left\{x \in C^{1}(\bar{Z}): x(z)=0\right.$ for all $\left.z \in \partial Z\right\}$. The Banach space $C_{0}^{1}(\bar{Z})$ is an ordered Banach space with order cone given by

$$
C_{0}^{1}(\bar{Z})_{+}=\left\{x \in C_{0}^{1}(\bar{Z}): x(z) \geq 0 \forall z \in \bar{Z}\right\} .
$$

We know that $\operatorname{int} C_{0}^{1}(\bar{Z})_{+} \neq \varnothing$ and in fact

$$
\operatorname{int}_{0}^{1}(\bar{Z})_{+}=\left\{x \in C_{0}^{1}(\bar{Z})_{+}: x(z)>0 \forall z \in Z \text { and } \frac{\partial x}{\partial n}(z)<0 \forall z \in \partial Z\right\} .
$$

By virtue of the strong maximum principle of Vázquez [23], we have $u_{1} \in \operatorname{int} C_{0}^{1}(\bar{Z})_{+}$.

Using the Lusternik-Schnirelmann theory, in addition to $\hat{\lambda}_{1}(m)>0$, we obtain a whole strictly increasing sequence $\left\{\widehat{\lambda}_{k}(m)\right\}_{k \geq 1} \subseteq \mathbb{R}_{+}$of eigenvalues of (2.5), such that $\widehat{\lambda}_{k}(m) \rightarrow+\infty$ as $k \rightarrow \infty$. These are the so-called "variational eigenvalues" of $\left(-\Delta_{p}, W_{0}^{1, p}(Z), m\right)$. When $p=2$ (linear case), then these are all the eigenvalues. For $p \neq 2$ (nonlinear case), we do not know if this is true. Nevertheless exploiting the fact that $\widehat{\lambda}_{1}(m)>0$ is isolated, we can define

$$
\hat{\lambda}_{2}^{*}(m)=\inf \left\{\hat{\jmath}: \hat{\jmath} \text { is an eigenvalue of }(2.5), \hat{\jmath} \neq \widehat{\lambda}_{1}(m)\right\}>\widehat{\lambda}_{1}(m) \text {. }
$$

Because the set of eigenvalues of (2.5) is closed, we see that $\hat{\lambda}_{2}^{*}(m)$ is an eigenvalue of $\left(-\Delta_{p}, W_{0}^{1, p}(Z), m\right)$. In fact we have $\hat{\lambda}_{2}^{*}(m)=\hat{\lambda}_{2}(m)$; that is, the second eigenvalue and the second variational eigenvalue of $\left(-\Delta_{p}, W_{0}^{1, p}(Z), m\right)$ coincide. Then for $\hat{\lambda}_{2}(m)$ we have a variational expression provided by the Lusternik-Schnirelmann theory. The eigenvalues $\hat{\lambda}_{1}(m)$ and $\hat{\lambda}_{2}(m)$ exhibit some monotonicity properties with respect to the weight function $m \in L^{\infty}(Z)_{+}$. More precisely, we have the following.

(a) If $m(z) \leq m^{\prime}(z)$ a.e. on $Z$ with strict inequality on a set of positive measure, then $\hat{\lambda}_{1}\left(m^{\prime}\right)<\widehat{\lambda}_{1}(m)$ (this is immediate from (2.6)).

(b) If $m(z)<m^{\prime}(z)$ a.e. on $Z$, then $\hat{\lambda}_{2}\left(m^{\prime}\right)<\widehat{\lambda}_{2}(m)$ (see Anane-Tsouli [24]).

If $m \equiv 1$, then we write $\hat{\lambda}_{1}(m)=\lambda_{1}$ and $\hat{\lambda}_{2}(m)=\lambda_{2}$. For $\lambda_{2}>0$, there is an alternative variational characterization, due to Cuesta et al. [25]; namely, if $\partial B_{1}^{L^{p}(Z)}=\left\{x \in L^{p}(Z):\|x\|_{p}=\right.$ $1\}, S=W_{0}^{1, p}(Z) \cap \partial B_{1}^{L^{p}(Z)}$, and $\Gamma_{0}=\left\{\gamma_{0} \in C([-1,1], S): \gamma_{0}(-1)=-u_{1}, \gamma_{0}(1)=u_{1}\right\}$, then

$$
\lambda_{2}=\inf _{\gamma_{0} \in \Gamma_{0}} \sup _{x \in \gamma_{0}([-1,1])}\|D x\|_{p}^{p}
$$

Finally we recall the notions of upper and of lower solutions for problem (1.1).

(a) A function $\bar{x} \in W^{1, p}(Z)$ with $\left.\bar{x}\right|_{\partial Z} \geq 0$ is an "upper solution" for problem (1.1), if

$$
\int_{Z}\|D \bar{x}(z)\|^{p-2}(D \bar{x}(z), D \psi(z))_{\mathbb{R}^{\mathbb{N}}} d z \geq \int_{Z} u(z) \psi(z) d z
$$

for all $\psi \in W_{0}^{1, p}(Z), \psi(z) \geq 0$ a.e. on $Z$ and for some $u \in L^{\eta}(Z), u(z) \in \partial j(z, \bar{x}(z))$ a.e. on $Z$ for some $1<\eta<p^{*}=N p /(N-p)$ if $N>p,+\infty$ if $N \leq p$. 
(b) A function $\underline{x} \in W^{1, p}(Z)$ with $\left.\underline{x}\right|_{\partial Z} \leq 0$ is a "lower solution" for problem (1.1), if

$$
\int_{Z}\|D \underline{x}(z)\|^{p-2}(D \underline{x}(z), D \psi(z))_{\mathbb{R}^{\mathbb{N}}} d z \leq \int_{Z} u(z) \psi(z) d z
$$

for all $\psi \in W_{0}^{1, p}(Z), \psi(z) \geq 0$ a.e. on $Z$ and for some $u \in L^{\eta}(Z), u(z) \in \partial j(z, \underline{x}(z))$ a.e. on $Z$ for some $1<\eta<p^{*}$.

\section{Solutions of Constant Sign}

In this section, we produce two nontrivial solutions of (1.1) which have constant sign. The first is positive and the second is negative. To do this, we will need the following hypotheses on the nonsmooth potential $j(z, x)$.

$H(j)_{1}: j: Z \times \mathbb{R} \rightarrow \mathbb{R}$ is a function such that $j(z, 0)=0$ a.e. on $Z, \partial j(z, 0)=\{0\}$ a.e. on $Z$, and

(i) for every $x \in \mathbb{R}, z \rightarrow j(z, x)$ is measurable;

(ii) for almost all $z \in Z, x \rightarrow j(z, x)$ is locally Lipschitz;

(iii) for almost all $z \in Z$, all $x \in \mathbb{R}$, and all $u \in \partial j(z, x)$, we have

$$
|u| \leq a(z)+c|x|^{p-1} \quad \text { with } a \in L^{\infty}(Z)_{+}, c>0 ;
$$

(iv) there exists $\theta \in L^{\infty}(Z)_{+}$satisfying $\theta(z) \leq \lambda_{1}$ a.e. on $Z$ with strict inequality on a set of positive measure, such that

$$
\limsup _{|x| \rightarrow \infty} \frac{u}{|x|^{p-2} x} \leq \theta(z)
$$

uniformly for almost all $z \in Z$ and all $u \in \partial j(z, x)$;

(v) there exist $\eta, \hat{\eta} \in L^{\infty}(Z)_{+}$satisfying $\lambda_{1} \leq \eta(z) \leq \widehat{\eta}(z)$ a.e. on $Z$, where the first inequality is strict on a set of positive measure, such that

$$
\eta(z) \leq \liminf _{x \rightarrow 0} \frac{u}{|x|^{p-2} x} \leq \limsup _{x \rightarrow 0} \frac{u}{|x|^{p-2} x} \leq \widehat{\eta}(z)
$$

uniformly for almost all $z \in Z$ and all $u \in \partial j(z, x)$;

(vi) for almost all $z \in Z$, all $x \in \mathbb{R}$, and all $u \in \partial j(z, x)$, we have $u x \geq 0$ (sign condition).

Remark 3.1. Hypotheses $H(j)_{1}$ (iv) and (v) are nonuniform nonresonance conditions at zero and at $\pm \infty$, respectively. Moreover, as we move from 0 to $\pm \infty$, the "slopes" $u /|x|^{p-2} x . u \in$ $\partial j(z, x)$ cross the first eigenvalue $\lambda_{1}>0$. So our framework incorporates the so-called asymptotically $p$-linear equations. For $p=2$, since the appearance of the pioneering work of Amann-Zehnder [26], these problems have attracted a lot of interest. 
The next lemma is an easy consequence of the strict positivity of $u_{1} \in C_{0}^{1}(\bar{Z})$ and of the hypotheses on $\theta \in L^{\infty}(Z)_{+}$(see $H(j)(i v)$ ). We omit the proof.

Lemma 3.2. If $\theta \in L^{\infty}(Z)_{+}$satisfies $\theta(z) \leq \lambda_{1}$ a.e. on $Z$ with strict inequality on a set of positive measure, then there exists $\xi_{0}>0$ such that

$$
\|D x\|_{p}^{p}-\int_{Z} \theta(z)|x(z)|^{p} d z \geq \xi_{0}\|D x\|_{p}^{p} \quad \forall x \in W_{0}^{1, p}(Z) .
$$
problem:

Given $\varepsilon>0$ and $\gamma_{\varepsilon} \in L^{\infty}(Z)_{+}, \gamma_{\varepsilon} \neq 0$, we consider the following nonlinear Dirichlet

$$
\begin{gathered}
-\operatorname{div}\left(\|D x(z)\|^{p-2} D x(z)\right)=(\theta(z)+\varepsilon)|x(z)|^{p-2} x(z)+\gamma_{\varepsilon}(z) \quad \text { a.e. on } Z, \\
\left.x\right|_{\partial Z}=0 .
\end{gathered}
$$

In the next proposition, we establish the solvability of (3.5).

Proposition 3.3. If $\theta \in L^{\infty}(Z)_{+}$satisfies $\theta \leq \lambda_{1}$ a.e. on $Z$ with strict inequality on a set of positive measure, then for all $\varepsilon>0$ small problem (3.5) admits a solution $\bar{x} \in \operatorname{int} C_{0}^{1}(\bar{Z})_{+}$.

Proof. In what follows by $\langle\cdot, \cdot\rangle$ we denote the duality brackets for the pair $\left(W^{-1, p^{\prime}}(Z), W_{0}^{1, p}(Z)\right)\left(1 / p+1 / p^{\prime}=1\right)$. We introduce the nonlinear operator $A: W_{0}^{1, p}(Z) \rightarrow$ $W^{-1, p^{\prime}}(Z)$ defined by

$$
\langle A(x), y\rangle=\int_{Z}\|D x(z)\|^{p-2}(D x(z), D y(z))_{\mathbb{R}^{\mathbb{N}}} d z \quad \forall x, y \in W_{0}^{1, p}(Z) .
$$

It is straightforward to check that $A$ is strictly monotone and demicontinuous, hence maximal monotone too. Also let $N_{\varepsilon}: L^{p}(Z) \rightarrow L^{p^{\prime}}(Z)$ be the nonlinear, bounded, continuous map defined by

$$
N_{\varepsilon}(x)(\cdot)=(\theta(\cdot)+\varepsilon)|x(\cdot)|^{p-2} x(\cdot) .
$$

Because of the compact embedding of $W_{0}^{1, p}(Z)$ into $L^{p}(Z), N_{\varepsilon}$ viewed as a map from $W_{0}^{1, p}(Z)$ into $L^{p^{\prime}}(Z)$ is completely continuous. Therefore $x \rightarrow G_{\varepsilon}(x)=A(x)-N_{\varepsilon}(x)$ is pseudomonotone from $W_{0}^{1, p}(Z)$ into $W^{-1, p^{\prime}}(Z)$. Also for every $x \in W_{0}^{1, p}(Z)$, we have

$$
\begin{aligned}
\left\langle G_{\varepsilon}(x), x\right\rangle & =\|D x\|_{p}^{p}-\int_{Z} \theta(z)|x(z)|^{p} d z-\varepsilon\|x\|_{p}^{p} \\
& \geq\left(\xi_{0}-\frac{\varepsilon}{\lambda_{1}}\right)\|D x\|_{p}^{p} \text { (see Lemma } 3.2 \text { and (2.6)). }
\end{aligned}
$$


Therefore, if $\varepsilon<\lambda_{1} \xi_{0}$, then by virtue of Poincare's inequality $G_{\varepsilon}(\cdot)$ is coercive. But a pseudomonotone coercive operator is surjective. Hence we can find that $\bar{x} \in W_{0}^{1, p}(Z)$ such that

$$
\begin{aligned}
& G_{\varepsilon}(\bar{x})=A(\bar{x})-N_{\varepsilon}(\bar{x})=\gamma_{\varepsilon}, \\
& \Longrightarrow\left\{\begin{array}{c}
-\operatorname{div}\left(\|D \bar{x}(z)\|^{p-2} D \bar{x}(z)\right)=(\theta(z)+\varepsilon)|\bar{x}(z)|^{p-2} \bar{x}(z)+\gamma_{\varepsilon}(z) \text { a.e. on } Z, \\
\left.\bar{x}\right|_{\partial Z}=0
\end{array}\right\} .
\end{aligned}
$$

Thus $\bar{x} \in W_{0}^{1, p}(Z)$ is a solution of (3.5). We take duality brackets of (3.9) with the test function $-\bar{x}^{-}=-\max \{-\bar{x}, 0\} \in W_{0}^{1, p}(Z)$. We obtain

$$
\begin{aligned}
& \left\|D \bar{x}^{-}\right\|_{p}^{p}-\int_{Z} \theta(z)\left|x^{-}(z)\right|^{p} d z \leq \varepsilon\left\|\bar{x}^{-}\right\|_{p}^{p} \quad\left(\text { since } \gamma_{\varepsilon} \geq 0\right) \\
& \Longrightarrow \xi_{0}\left\|D \bar{x}^{-}\right\|_{p}^{p} \leq \frac{\varepsilon}{\lambda_{1}}\left\|D \bar{x}^{-}\right\|_{p}^{p} \quad \text { (see Lemma } 3.2 \text { and (2.6)). }
\end{aligned}
$$

But recall that $\varepsilon<\lambda_{1} \xi_{0}$. So it follows that $\|D \bar{x}\|_{p}=0$, hence $\bar{x}^{-}=0$; that is, $\bar{x} \geq 0$. Since $\gamma_{\varepsilon} \neq 0$, from (3.10) it follows that $\bar{x} \neq 0$ and $\bar{x} \in C_{0}^{1}(\bar{Z})$ (nonlinear regularity theory). In addition, from (3.10), we see that

$$
\begin{aligned}
& \operatorname{div}\left(\|D \bar{x}(z)\|^{p-2} D \bar{x}(z)\right) \leq 0 \quad \text { a.e. on } Z, \\
& \Longrightarrow \bar{x} \in \operatorname{int} C_{0}^{1}(\bar{Z})_{+} \quad(\text { see Vázquez [23]). }
\end{aligned}
$$

In fact the solution $\bar{x} \in \operatorname{int} C_{0}^{1}(\bar{Z})_{+}$of (3.5) is an upper solution for problem (1.1).

Proposition 3.4. If hypotheses $H(j)_{1}(i) \rightarrow(i v)$ hold and $\varepsilon>0$ is small, then the solution $\bar{x} \in$ int $C_{0}^{1}(\bar{Z})_{+}$of problem (3.5) obtained in Proposition 3.3 is a strict upper solution of problem (1.1) (strict means that $\bar{x}$ is an upper solution of (1.1) which is not a solution).

Proof. Because of hypotheses $H(j)_{1}(\mathrm{iv})$, given $\varepsilon>0$, we can find $M_{1}=M_{1}(\varepsilon)>0$ such that for almost all $z \in Z$, all $x \geq M_{1}$, and all $u \in \partial j(z, x)$, we have

$$
u \leq(\theta(z)+\varepsilon) x^{p-1}
$$

Also due to hypothesis $H(j)_{1}$ (iii), we can find $\gamma_{\varepsilon} \in L^{\infty}(Z)_{+}, \gamma_{\varepsilon} \neq 0$ such that for almost all $z \in Z$, all $x \in\left[0, M_{1}\right]$, and all $u \in \partial j(z, x)$, we have

$$
u<\gamma_{\varepsilon}(z)
$$

Therefore it follows that for almost all $z \in Z$, all $x \geq 0$, and all $u \in \partial j(z, x)$, we have

$$
u<(\theta(z)+\varepsilon) x^{p-1}+\gamma_{\varepsilon}(z)
$$


So for $0<\varepsilon<\lambda_{1} \xi_{0}$ and $\gamma_{\varepsilon}$ as above, we consider problem (3.5). From Proposition 3.3, we have a solution $\bar{x} \in \operatorname{int} C_{0}^{1}(\bar{Z})_{+}$. Then due to (3.15), for all $u \in L^{p^{\prime}}(Z)_{+}$with $u(z) \in$ $\partial j(z, \bar{x}(z))$ a.e. on $Z$, we have

$$
\begin{aligned}
& u(z)<(\theta(z)+\varepsilon) \bar{x}(z)^{p-1}+\gamma_{\varepsilon}(z) \text { a.e. on } Z \\
& \Longrightarrow \bar{x} \in \operatorname{int} C_{0}^{1}(\bar{Z})_{+} \text {is a strict upper solution for problem (1.1). }
\end{aligned}
$$

Since $\partial j(z, 0)=\{0\}$ a.e. on $Z, \underline{x} \equiv 0$ is a lower solution for problem (1.1).

We introduce the set

$$
C=\left\{x \in W_{0}^{1, p}(Z): 0 \leq x(z) \leq \bar{x}(z) \text { a.e. on } Z\right\}
$$

and the truncation function $\tau_{+}: \mathbb{R} \rightarrow \mathbb{R}_{+}$defined by

$$
\tau_{+}(x)= \begin{cases}0 & \text { if } x \leq 0 \\ x & \text { if } x>0\end{cases}
$$

Then we set $j_{+}(\cdot, x)=j\left(z, \tau_{+}(x)\right)$ and we consider the locally Lipschitz functional $\varphi_{+}$: $W_{0}^{1, p}(Z) \rightarrow \mathbb{R}$ defined by

$$
\varphi_{+}(x)=\frac{1}{p}\|D x\|_{p}^{p}-\int_{Z} j_{+}(z, x(z)) d z \quad \forall x \in W_{0}^{1, p}(Z) .
$$

We will show that we can find a nontrivial solution of (1.1) in $C$, which is a local minimizer of $\varphi_{+}$and of $\varphi$. To do this we will need the following simple result about ordered Banach spaces.

Lemma 3.5. If $X$ is an ordered Banach space, $K$ is the order cone of $X, \operatorname{int} K \neq \varnothing$, and $x_{0} \in \operatorname{int} K$, then for every $y \in X$, we can find $t=t(y)>0$ such that $t x_{0}-y \in \operatorname{int} K$.

Proof. Since $x_{0} \in \operatorname{int} K$, we can find $\delta>0$ such that

$$
\bar{B}_{\delta}\left(x_{0}\right)=\left\{x \in X:\left\|x-x_{0}\right\| \leq \delta\right\} \subseteq \operatorname{int} K .
$$

Let $y \in X, y \neq 0$ (if $y=0$, then clearly the lemma holds for all $t>0$ ). We have the following:

$$
\begin{aligned}
& x_{0}-\delta \frac{y}{\|y\|} \in \operatorname{int} K, \\
& \Longrightarrow \frac{\|y\|}{\delta} x_{0}-y \in \operatorname{int} K .
\end{aligned}
$$

So, if $t=\|y\| / \delta$, then $t x_{0}-y \in \operatorname{int} K$. 
Using this lemma, we can prove the following result.

Proposition 3.6. If hypotheses $H(j)_{1}$ hold, then there exists $x_{0} \in C$ which is a local minimizer of $\varphi_{+}$ and of $\varphi$.

Proof. From (3.15), we know that given $\varepsilon>0$, we can find $\gamma_{\varepsilon} \in L^{\infty}(Z)_{+}, \gamma_{\varepsilon} \neq 0$ such that

$$
\begin{array}{r}
u<(\theta(z)+\varepsilon) x^{p-1}+\gamma_{\varepsilon}(z) \text { for a.a. } z \in Z, \text { all } x \geq 0, \\
\text { and all } u \in \partial j_{+}(z, x)=\partial j(z, x) .
\end{array}
$$

Because of hypotheses $H(j)_{1}(\mathrm{i})$, (ii), for almost all $z \in Z, x \rightarrow j_{+}(z, x)$ is almost everywhere differentiable on $\mathbb{R}$ (Rademacher's theorem) and at every point of differentiability we have

$$
\begin{aligned}
& \frac{d}{d x} j_{+}(z, x) \in \partial j_{+}(z, x) \\
& \Longrightarrow \frac{d}{d x} j_{+}(z, x)<(\theta(z)+\varepsilon) x^{p-1}+\gamma_{\varepsilon}(z) \quad \text { for a.a. } z \in Z \text {, all } x \geq 0 \text { (see (3.22)). }
\end{aligned}
$$

Integrating this inequality and since $\left.j_{+}(z, x)\right|_{\mathbb{R}_{-}}=0$ for almost all $z \in Z$, we obtain

$$
j_{+}(z, x)<\frac{1}{p}(\theta(z)+\varepsilon)|x|^{p}+\gamma_{\varepsilon}(z)|x| \quad \text { for a.a. } z \in Z \text {, all } x \in \mathbb{R} .
$$

Then for every $x \in W_{0}^{1, p}(Z)$, we have

$$
\begin{aligned}
\varphi_{+}(x) & =\frac{1}{p}\|D x\|_{p}^{p}-\int_{Z} j_{+}(z, x(z)) d z \\
& >\frac{1}{p}\|D x\|_{p}^{p}-\frac{1}{p} \int_{Z} \theta(z)|x(z)|^{p} d z-\frac{\varepsilon}{p}\|x\|_{p}^{p}-c_{1}\|D x\|_{p} \\
& \quad \text { for some } c_{1}>0 \text { (see (3.24)) } \\
& \geq \frac{1}{p}\left(\xi_{0}-\frac{\varepsilon}{\lambda_{1}}\right)\|D x\|_{p}^{p}-c_{1}\|D x\|_{p} \text { (see Lemma 3.2). }
\end{aligned}
$$

Choosing $\varepsilon<\lambda_{1} \xi_{0}$, because $p>1$, from (3.25) and Poincare's inequality, we infer that $\varphi_{+}$is coercive. Also it is easy to see that $\varphi_{+}$is weakly lower semicontinuous on $W_{0}^{1, p}(Z)$. Hence by virtue of the theorem of Weierstrass, we can find $x_{0} \in C$ such that

$$
\varphi_{+}\left(x_{0}\right)=\inf _{C} \varphi_{+}
$$

First we show that $x_{0} \neq 0$. To this end, note that hypothesis $H(j)_{1}(\mathrm{v})$ implies that given $\varepsilon>0$, we can find $\delta=\delta(\varepsilon)>0$ such that

$$
u \geq(\eta(z)-\varepsilon) x^{p-1} \quad \text { for a.a. } z \in Z \text {, all } x \in[0, \delta] \text { and all } u \in \partial j_{+}(z, x)=\partial j(z, x) .
$$


As before, integrating (3.27), we obtain

$$
j_{+}(z, x) \geq \frac{1}{p}(\eta(z)-\varepsilon) x^{p} \quad \text { for a.a. } z \in Z, \text { all } x \in[0, \delta] .
$$

We know that $\bar{x} \in \operatorname{int} C_{0}^{1}(\bar{Z})_{+}$(see Proposition 3.3). So using Lemma 3.5, we can find $\mu>0$ small such that

$$
\mu u_{1}(z) \leq \min \{\bar{x}(z), \delta\} \quad \forall z \in \bar{Z}
$$

Then, because of (3.28), we have

$$
\begin{aligned}
\varphi_{+}\left(\mu u_{1}\right) & =\frac{\mu^{p}}{p}\left\|D u_{1}\right\|_{p}^{p}-\int_{Z} j_{+}\left(z, \mu u_{1}(z)\right) d z \\
& \leq \frac{\mu^{p}}{p}\left\|D u_{1}\right\|_{p}^{p}-\frac{\mu^{p}}{p} \int_{Z} \eta(z) u_{1}(z)^{p} d z+\frac{\mu^{p} \varepsilon}{p}\left\|u_{1}\right\|_{p}^{p} \\
& =\frac{\mu^{p}}{p}\left[\int_{Z}\left(\lambda_{1}-\eta(z)\right) u_{1}(z)^{p} d z+\varepsilon\left\|u_{1}\right\|_{p}^{p}\right]
\end{aligned}
$$

Let $\sigma=\int_{Z}\left(\lambda_{1}-\eta(z)\right) u_{1}(z)^{p} d z$. Using the hypothesis on $\eta$ (see $\left.H(j)_{1}(\mathrm{v})\right)$ and the fact that $u_{1}(z)>0$ for all $z \in Z$, we see that $\sigma<0$. So, if we choose $\varepsilon<-\sigma /\left\|u_{1}\right\|_{p}^{p}$, we have

$$
\varphi_{+}\left(\mu u_{1}\right)<0 \quad \forall \mu>0 \text { small. }
$$

Note that for $\mu>0$ small, $\mu u_{1} \in C$. Hence

$$
\begin{aligned}
& \varphi_{+}\left(x_{0}\right)=\inf _{C} \varphi_{+} \leq \varphi_{+}\left(\mu u_{1}\right)<0=\varphi_{+}(0) \quad(\text { see }(3.31)), \\
& \Longrightarrow x_{0} \neq 0, \quad x_{0} \in C .
\end{aligned}
$$

Given any $y \in C$, we define $k_{0}(t)=\varphi_{+}\left(t y+(1-t) x_{0}\right), t \in[0,1]$. Then $k$ is Lipschitz continuous, hence differentiable almost everywhere and $k_{0}(0) \leq k_{0}(t)$ for all $t \in[0,1]$. From Chang [27, page 106], we know that we can find $u \in L^{p^{\prime}}(Z), u(z) \in \partial j_{+}\left(z, x_{0}(z)\right)=$ $\partial j\left(z, x_{0}(z)\right)$ a.e. on $Z$, such that

$$
0 \leq\left\langle A\left(x_{0}\right), y-x_{0}\right\rangle-\int_{Z} u(z)\left(y-x_{0}\right)(z) d z, \quad \forall y \in C
$$

For any $v \in W_{0}^{1, p}(Z)$ and $\varepsilon>0$, we define

$$
y(z)= \begin{cases}0 & \text { if } z \in\left\{x_{0}+\varepsilon v \leq 0\right\}, \\ x_{0}(z)+\varepsilon v(z) & \text { if } z \in\left\{0<x_{0}+\varepsilon v<\bar{x}\right\}, \\ \bar{x}(z) & \text { if } z \in\left\{\bar{x} \leq x_{0}+\varepsilon v\right\} .\end{cases}
$$


Clearly $y \in C$. We use this $y \in C$ in (3.33). Hence we obtain

$$
\begin{aligned}
0 \leq & \varepsilon \int_{\left\{0<x_{0}+\varepsilon v<\bar{x}\right\}}\left\|D x_{0}\right\|^{p-2}\left(D x_{0}, D v\right)_{\mathbb{R}^{\mathbb{N}}} d z-\int_{\left\{0<x_{0}+\varepsilon v<\bar{x}\right\}} u(\varepsilon v) d z \\
& -\int_{\left\{x_{0}+\varepsilon v \leq 0\right\}}\left\|D x_{0}\right\|^{p} d z+\int_{\left\{x_{0}+\varepsilon v \leq 0\right\}} u x_{0} d z \\
& +\int_{\left\{x_{0}+\varepsilon v \geq \bar{x}\right\}}\left\|D x_{0}\right\|^{p-2}\left(D x_{0}, D\left(\bar{x}-x_{0}\right)\right)_{\mathbb{R}^{\mathbb{N}}} d z-\int_{\left\{x_{0}+\varepsilon v \geq \bar{x}\right\}} u\left(\bar{x}-x_{0}\right) d z \\
= & \varepsilon \int_{Z}\left\|D x_{0}\right\|^{p-2}\left(D x_{0}, D v\right)_{\mathbb{R}^{\mathbb{N}}} d z-\varepsilon \int_{Z} u v d z \\
& -\int_{\left\{x_{0}+\varepsilon v \geq \bar{x}\right\}}\|D \bar{x}\|^{p-2}\left(D \bar{x}, D\left(x_{0}+\varepsilon v-\bar{x}\right)_{\mathbb{R}^{\mathbb{N}}} d z+\int_{\left\{x_{0}+\varepsilon v \geq \bar{x}\right\}} \bar{u}\left(x_{0}+\varepsilon v-\bar{x}\right) d z\right.
\end{aligned}
$$

$\left(\bar{u} \in L^{p^{\prime}}(Z), \bar{u}(z) \in \partial j_{+}(z, \bar{x}(z))\right.$ a.e. and clearly from the definition of $\bar{u}$, we can always assume $\bar{u}=u$ a.e. on $\left\{x=x_{0}\right\}$ )

$$
\begin{aligned}
& +\int_{\left\{x_{0}+\varepsilon v \leq 0\right\}} u\left(x_{0}+\varepsilon v\right) d z+\int_{\left\{x_{0}+\varepsilon v \geq \bar{x}\right\}}(\bar{u}-u)\left(\bar{x}-x_{0}-\varepsilon v\right) d z \\
& -\int_{\left\{x_{0}+\varepsilon v \leq 0\right\}}\left\|D x_{0}\right\|^{p} d z-\varepsilon \int_{\left\{x_{0}+\varepsilon v \leq 0\right\}}\left\|D x_{0}\right\|^{p-2}\left(D x_{0}, D v\right)_{\mathbb{R}^{\mathbb{N}}} d z \\
& +\int_{\left\{x_{0}+\varepsilon v \geq \bar{x}\right\}}\left(\|D \bar{x}\|^{p-2} D \bar{x}-\left\|D x_{0}\right\|^{p-2} D x_{0}, D\left(x_{0}-\bar{x}\right)\right)_{\mathbb{R}^{\mathbb{N}}} d z \\
& +\varepsilon \int_{\left\{x_{0}+\varepsilon v \geq \bar{x}\right\}}\left(\|D \bar{x}\|^{p-2} D \bar{x}-\left\|D x_{0}\right\|^{p-2} D x_{0}, D v\right)_{\mathbb{R}^{\mathbb{N}}} d z .
\end{aligned}
$$

Using $h=\left(x_{0}+\varepsilon v-\bar{x}\right)^{+} \in W_{0}^{1, p}(Z)_{+}$as a test function, from the definition of an upper solution for problem (1.1), we have

$$
-\int_{\left\{x_{0}+\varepsilon v \geq \bar{x}\right\}}\|D \bar{x}\|^{p-2}\left(D \bar{x}, D\left(x_{0}+\varepsilon v-\bar{x}\right)\right)_{\mathbb{R}^{\mathbb{N}}} d z+\int_{\left\{x_{0}+\varepsilon v \geq \bar{x}\right\}} \bar{u}\left(x_{0}+\varepsilon v-\bar{x}\right) d z \leq 0 .
$$

Also from the (strict) monotonicity of the operator $A$, we have

$$
\int_{\left\{x_{0}+\varepsilon v \geq \bar{x}\right\}}\left(\|D \bar{x}\|^{p-2} D \bar{x}-\left\|D x_{0}\right\|^{p-2} D x_{0}, D\left(x_{0}-\bar{x}\right)\right)_{\mathbb{R}^{\mathbb{N}}} d z \leq 0 .
$$

From hypothesis $H(j)_{1}(v i)$, it follows that

$$
\int_{\left\{x_{0}+\varepsilon v \leq 0\right\}} u\left(x_{0}+\varepsilon v\right) d z \leq 0 .
$$


Since $x_{0} \in C_{0}^{1}(\bar{Z})$, we have

$$
\begin{aligned}
& \int_{\left\{x_{0}+\varepsilon v \geq \bar{x}\right\}}(\bar{u}-u)\left(\bar{x}-x_{0}-\varepsilon v\right) d z \\
& \quad=\int_{\left\{x_{0}+\varepsilon v \geq \bar{x}>x_{0}\right\}}(\bar{u}-u)\left(\bar{x}-x_{0}-\varepsilon v\right) d z \\
& \left.\quad \text { (recall that } \bar{u}=u \text { a.e. on }\left\{\bar{x}=x_{0}\right\}, x_{0} \leq \bar{x} \text { since } x_{0} \in C\right) \\
& \quad \leq c_{2} \int_{\left\{x_{0}+\varepsilon v \geq \bar{x}>x_{0}\right\}}\left(x_{0}+\varepsilon v-\bar{x}\right) d z \text { for some } c_{2}>0 \\
& \quad \leq \varepsilon c_{2} \int_{\left\{x_{0}+\varepsilon v \geq \bar{x}>x_{0}\right\}} v d z\left(\text { since } x_{0} \leq \bar{x}\right) .
\end{aligned}
$$

Returning to (3.35) and using (3.36) $\rightarrow$ (3.39), we obtain

$$
\begin{aligned}
0 \leq & \varepsilon \int_{Z}\left\|D x_{0}\right\|^{p-2}\left(D x_{0}, D v\right)_{\mathbb{R}^{\mathbb{N}}} d z-\varepsilon \int_{Z} u v d z \\
& +\varepsilon c_{2} \int_{\left\{x_{0}+\varepsilon v \geq \bar{x}>x_{0}\right\}} v d z-\varepsilon \int_{\left\{x_{0}+\varepsilon v \leq 0\right\}}\left\|D x_{0}\right\|^{p-2}\left(D x_{0}, D v\right)_{\mathbb{R}^{\mathbb{N}}} d z \\
& +\varepsilon \int_{\left\{x_{0}+\varepsilon v \geq \bar{x}\right\}}\left(\|D \bar{x}\|^{p-2} D \bar{x}-\left\|D x_{0}\right\|^{p-2} D x_{0}, D v\right)_{\mathbb{R}^{\mathbb{N}}} d z .
\end{aligned}
$$

We denote by $|\cdot|_{N}$ the Lebesgue measure on $\mathbb{R}^{\mathbb{N}}$. Then

$$
\left|\left\{x_{0}+\varepsilon v \geq \bar{x}>x_{0}\right\}\right|_{N} \downarrow 0 \quad \text { as } \varepsilon \downarrow 0 .
$$

Moreover, from Stampacchia's theorem, we know that

$$
D x_{0}(z)=0 \quad \text { a.e. on }\left\{x_{0}=0\right\}, \quad D x_{0}(z)=D \bar{x}(z) \text { a.e. on }\left\{x_{0}=\bar{x}\right\} \text {. }
$$

If we divide (3.40) by $\varepsilon>0$ and then we pass to the limit as $\varepsilon \downarrow 0$, because of (3.41) and (3.42), we obtain

$$
0 \leq\left\langle A\left(x_{0}\right), v\right\rangle-\int_{Z} u v d z=\left\langle A\left(x_{0}\right)-u, v\right\rangle
$$

Recall that $v \in W_{0}^{1, p}(Z)$ was arbitrary. So from (3.43), it follows that

$$
\begin{aligned}
& A\left(x_{0}\right)=u, \\
& \Longrightarrow x_{0} \in W_{0}^{1, p}(Z) \text { is a solution of problem (1.1). }
\end{aligned}
$$


The nonlinear regularity theory implies that $x_{0} \in C_{0}^{1}(\bar{Z})$ and then since $x_{0} \neq 0, x_{0} \geq 0$ from the nonlinear strong maximum principle of Vázquez [23], we have $x_{0} \in \operatorname{int} C_{0}^{1}(\bar{Z})_{+}$.

From (3.22), we know that

$$
\left.u(z)<(\theta(z)+\varepsilon) \bar{x}(z)^{p-1}+\gamma_{\varepsilon}(z) \text { a.e. on } Z \text { (recall that } x_{0} \leq \bar{x}\right)
$$

Then Proposition 2.2 of Guedda-Véron [28] implies that

$$
\begin{aligned}
& x_{0}(z)<\bar{x}(z) \quad \forall z \in Z, \quad \frac{\partial \bar{x}}{\partial n}(z)<\frac{\partial x_{0}}{\partial n}(z) \quad \forall z \in \partial Z, \\
& \Longrightarrow \bar{x}-x_{0} \in \operatorname{int} C_{0}^{1}(\bar{Z})_{+} .
\end{aligned}
$$

Recall also that $x_{0} \in \operatorname{int} C_{0}^{1}(\bar{Z})_{+}$. Thus we can find $\delta>0$ such that

$$
\begin{gathered}
B_{\delta}^{C_{0}^{1}(\bar{Z})}\left(\bar{x}-x_{0}\right)=\left\{y \in C_{0}^{1}(\bar{Z}):\left\|y-\left(\bar{x}-x_{0}\right)\right\|_{C_{0}^{1}(\bar{Z})}<\delta\right\} \subseteq \operatorname{int} C_{0}^{1}(\bar{Z})_{+} \\
B_{\delta}^{C_{0}^{1}(\bar{Z})}\left(x_{0}\right)=\left\{y \in C_{0}^{1}(\bar{Z}):\left\|y-x_{0}\right\|_{C_{0}^{1}(\bar{Z})}<\delta\right\} \subseteq \operatorname{int} C_{0}^{1}(\bar{Z})_{+}
\end{gathered}
$$

These inclusions imply that

$$
\bar{x}-\left(x_{0}+B_{\delta}^{C_{0}^{1}(\bar{Z})}\right) \subseteq \operatorname{int} C_{0}^{1}(\bar{Z})_{+}, \quad x_{0}+B_{\delta}^{C_{0}^{1}(\bar{Z})} \subseteq \operatorname{int} C_{0}^{1}(\bar{Z})_{+} .
$$

The solution $x_{0}$ was obtained as a minimizer of $\varphi_{+}$on $C$. Then (3.48) implies that $x_{0}$ is also a local minimizer of $\varphi_{+}$and of $\varphi$ on $C_{0}^{1}(\bar{Z})$. But then from Motreanu-Papageorgiou [29] (see also Gasiński -Papageorgiou [21, pages 655-656]), it follows that $x_{0}$ is also a local $W_{0}^{1, p}(Z)$-minimizer of $\varphi_{+}$and of $\varphi$ too.

So far we have worked on the positive semiaxis. Next we repeat the same analysis on the negative semiaxis. More precisely, given $\varepsilon>0$ and $\gamma_{\varepsilon} \in L^{\infty}(Z)_{+}, \gamma_{\varepsilon} \neq 0$, we consider the following auxiliary problem:

$$
\begin{gathered}
-\operatorname{div}\left(\|D v(z)\|^{p-2} D v(z)\right)=(\theta(z)+\varepsilon)|v(z)|^{p-2} v(z)-\gamma_{\varepsilon}(z) \quad \text { a.e. on } Z \\
\left.v\right|_{\partial Z}=0 .
\end{gathered}
$$

Then as in the proof of Proposition 3.3, through the surjectivity of the pseudomonotone coercive operator $v \rightarrow G_{\varepsilon}(v)=A(v)-N_{\varepsilon}(v)$, we obtain a solution $\underline{u} \in-\operatorname{int} C_{0}^{1}(\bar{Z})_{+}$of (3.49). We can check that $\underline{v}$ is a strict lower solution of (1.1), while clearly $\bar{v} \equiv 0$ is an upper solution (in fact a solution) of (1.1). This time we consider the set

$$
D=\left\{v \in W_{0}^{1, p}(Z): \underline{v}(z) \leq v(z) \leq 0 \text { a.e. on } Z\right\}
$$


and the truncation function $\tau_{-}: \mathbb{R} \rightarrow \mathbb{R}_{-}$defined by

$$
\tau_{-}(x)= \begin{cases}x & \text { if } x<0 \\ 0 & \text { if } x \geq 0\end{cases}
$$

We set $j_{-}(z, x)=j\left(z, \tau_{-}(x)\right)$ and then introduce the locally Lipschitz functional $\varphi_{-}$: $W_{0}^{1, p}(Z) \rightarrow \mathbb{R}$ defined by

$$
\varphi_{-}(x)=\frac{1}{p}\|D x\|_{p}^{p}-\int_{Z} j_{-}(z, x(z)) d z \quad \forall x \in W_{0}^{1, p}(Z) .
$$

We consider the minimization problem

$$
\inf _{D} \varphi_{-}
$$

Arguing as in Proposition 3.3, we obtain the following.

Proposition 3.7. If hypotheses $H(j)_{1}$ hold, then there exists $v_{0} \in D$ which is a local minimizer of $\varphi_{-}$ and of $\varphi$.

Now combining Propositions 3.6 and 3.7, we obtain a multiplicity result for problem (1.1) with solutions of constant sign.

Theorem 3.8. If hypotheses $H(j)_{1}$ hold, then the problem (1.1) has at least two solutions $x_{0} \in$ $\operatorname{int} C_{0}^{1}(\bar{Z})_{+}$and $v_{0} \in-\operatorname{int} C_{0}^{1}(\bar{Z})_{+}$.

Remark 3.9. From Propositions 3.6 and 3.7, we know that both $x_{0}$ and $v_{0}$ are local minimizers of $\varphi$. So we must have a third critical point of $\varphi$, distinct from $x_{0}, v_{0}$. However, at this point we cannot guarantee that it is nontrivial. In the next section by strengthening our hypothesis on $j(z, \cdot)$ near the origin (see $\left.H(j)_{1}(\mathrm{v})\right)$, we will be able to show that this third critical point is nontrivial and in fact is a nodal solution.

\section{Existence of Nodal Solution}

Recall that every eigenfunction of (2.5) corresponding to an eigenvalue $\widehat{\lambda} \neq \widehat{\lambda}_{1}$ must change sign. So we expect that in general the sign changing solutions of (1.1) must be more than the solutions of constant sign. Nevertheless to produce a sign-changing solution (also known as nodal solution) for (1.1) is a rather involved process.

Here we follow an approach first employed by Dancer-Du [6] for semilinear problems (i.e., $p=2$ ) and recently extended to problems with the $p$-Laplacian and a smooth potential by Carl-Perera [8]. Roughly speaking, the strategy is as follows. Continuing with the argument used in Section 3, we produce the smallest positive solution $y_{+}$and the largest negative solution $y_{-}$. Then we form order interval $\left[y_{-}, y_{+}\right]$. Using variational techniques (in particular Theorem 2.2) we produce a solution $y_{0}$ of $(1.1)$ in $\left[y_{-}, y_{+}\right]$different from $y_{-}$and $y_{+}$. Evidently if $y_{0} \neq 0$, then $y_{0}$ must be sign changing. To show that $y_{0}$ is nontrivial, we employ Theorem 2.5 and (2.10). In addition to the works of Dancer-Du [6] and Carl-Perera [8], variants of this 
method can also be found in the works of Ambrosetti-Garcia Azorero-Peral Alonso [3] and Jin [30]. A different approach based on the construction of a pseudogradient vector field with appropriate invariance properties can be found in Zhang-Li [7, 9], Zhang et al. [10] (see also Li-Wang [31]).

We start executing the solution strategy outlined above by proving first a lemma which establishes that the set of upper solutions for problem (1.1) is downward directed.

Lemma 4.1. If $y_{1}, y_{2} \in W^{1, p}(Z)$ are two upper solutions for problem (1.1) and $y=\min \left\{y_{1}, y_{2}\right\} \in$ $W^{1, p}(Z)$, then $y$ is also an upper solution for problem (1.1).

Proof. Given $\varepsilon>0$, we consider the truncation function $\xi_{\varepsilon}: \mathbb{R} \rightarrow \mathbb{R}$ defined by

$$
\xi_{\varepsilon}(s)= \begin{cases}\varepsilon & \text { if } s \geq \varepsilon \\ s & \text { if } s \in[-\varepsilon, \varepsilon] \\ -\varepsilon & \text { if } s \leq-\varepsilon\end{cases}
$$

Clearly $\xi_{\varepsilon}$ is Lipschitz continuous. So from Marcus-Mizel [32], we have

$$
\begin{gathered}
\xi_{\varepsilon}\left(\left(y_{1}-y_{2}\right)^{-}\right) \in W^{1, p}(Z) \\
D \xi_{\varepsilon}\left(\left(y_{1}-y_{2}\right)^{-}\right)=\xi_{\varepsilon}^{\prime}\left(\left(y_{1}-y_{2}\right)^{-}\right) D\left(y_{1}-y_{2}\right)^{-} .
\end{gathered}
$$

Consider a test function $\psi \in C_{c}^{1}(Z)$ with $\psi \geq 0$. Then

$$
\begin{gathered}
\xi_{\varepsilon}\left(\left(y_{1}-y_{2}\right)^{-}\right) \psi \in W^{1, p}(Z) \cap L^{\infty}(Z), \\
D\left(\xi_{\varepsilon}\left(\left(y_{1}-y_{2}\right)^{-}\right) \psi\right)=\psi D \xi_{\varepsilon}\left(\left(y_{1}-y_{2}\right)^{-}\right)+\xi_{\varepsilon}\left(\left(y_{1}-y_{2}\right)^{-}\right) D \psi .
\end{gathered}
$$

Because $y_{1}, y_{2} \in W^{1, p}(Z)$ are upper solutions for problem (1.1), we have

$$
\begin{gathered}
\left\langle A\left(y_{1}\right), \xi_{\varepsilon}\left(\left(y_{1}-y_{2}\right)^{-}\right) \psi\right\rangle \geq\left\langle u_{1}, \xi_{\varepsilon}\left(\left(y_{1}-y_{2}\right)^{-}\right) \psi\right\rangle, \\
\left\langle A\left(y_{2}\right),\left(\varepsilon-\xi_{\varepsilon}\left(\left(y_{1}-y_{2}\right)^{-}\right)\right) \psi\right\rangle \geq\left\langle u_{2},\left(\varepsilon-\xi_{\varepsilon}\left(\left(y_{1}-y_{2}\right)^{-}\right)\right) \psi\right\rangle
\end{gathered}
$$

for some $u_{k} \in L^{p^{\prime}}(Z)$ with $u_{k}(z) \in \partial j\left(z, y_{k}(z)\right)$ a.e. on $Z, k=1$, 2. Adding these inequalities, we obtain

$$
\begin{array}{r}
\left\langle A\left(y_{1}\right), \xi_{\varepsilon}\left(\left(y_{1}-y_{2}\right)^{-}\right) \psi\right\rangle+\left\langle A\left(y_{2}\right),\left(\varepsilon-\xi_{\varepsilon}\left(\left(y_{1}-y_{2}\right)^{-}\right) \psi\right\rangle\right. \\
\geq\left\langle u_{1}, \xi_{\varepsilon}\left(\left(y_{1}-y_{2}\right)^{-}\right) \psi\right\rangle+\left\langle u_{2},\left(\varepsilon-\xi_{\varepsilon}\left(\left(y_{1}-y_{2}\right)^{-}\right) \psi\right\rangle\right.
\end{array}
$$


Note that

$$
\begin{aligned}
&\left\langle A\left(y_{1}\right), \xi_{\varepsilon}\left(\left(y_{1}-y_{2}\right)^{-}\right) \psi\right\rangle \\
&= \int_{Z}\left\|D y_{1}\right\|^{p-2}\left(D y_{1}, D\left(y_{1}-y_{2}\right)^{-}\right)_{\mathbb{R}^{\mathbb{N}}} \xi_{\varepsilon}^{\prime}\left(\left(y_{1}-y_{2}\right)^{-}\right) \psi d z \\
&+\int_{Z}\left\|D y_{1}\right\|^{p-2}\left(D y_{1}, D \psi\right)_{\mathbb{R}^{\mathbb{N}}} \xi_{\varepsilon}\left(\left(y_{1}-y_{2}\right)^{-}\right) d z \\
&=-\int_{\left\{-\varepsilon \leq y_{1}-y_{2} \leq 0\right\}}\left\|D y_{1}\right\|^{p-2}\left(D y_{1}, D\left(y_{1}-y_{2}\right)\right)_{\mathbb{R}^{\mathbb{N}}} \psi d z \\
&+\int_{Z}\left\|D y_{1}\right\|^{p-2}\left(D y_{1}, D \psi\right)_{\mathbb{R}^{\mathbb{N}}} \xi_{\varepsilon}\left(\left(y_{1}-y_{2}\right)^{-}\right) d z \\
&\left\langle A\left(y_{2}\right),\left(\varepsilon-\xi_{\varepsilon}\left(\left(y_{1}-y_{2}\right)^{-}\right)\right) \psi\right\rangle \\
&=\int_{\left\{-\varepsilon \leq y_{1}-y_{2} \leq 0\right\}}\left\|D y_{2}\right\|^{p-2}\left(D y_{2}, D\left(y_{1}-y_{2}\right)\right)_{\mathbb{R}^{\mathbb{N}}} \psi d z \\
& \quad+\int_{Z}\left\|D y_{2}\right\|^{p-2}\left(D y_{2}, D \psi\right)_{\mathbb{R}^{\mathbb{N}}}\left(\varepsilon-\xi_{\varepsilon}\left(\left(y_{1}-y_{2}\right)^{-}\right)\right) d z .
\end{aligned}
$$

Adding (4.6) and recalling that $\psi \geq 0$, we obtain

$$
\begin{aligned}
& \left\langle A\left(y_{1}\right), \xi_{\varepsilon}\left(\left(y_{1}-y_{2}\right)^{-}\right) \psi\right\rangle+\left\langle A\left(y_{2}\right),\left(\varepsilon-\xi_{\varepsilon}\left(\left(y_{1}-y_{2}\right)^{-}\right)\right) \psi\right\rangle \\
& =\int_{\left\{-\varepsilon \leq y_{1}-y_{2} \leq 0\right\}}\left(\left\|D y_{2}\right\|^{p-2} D y_{2}-\left\|D y_{1}\right\|^{p-2} D y_{1}, D\left(y_{1}-y_{2}\right)\right)_{\mathbb{R}^{\mathbb{N}}} \psi d z \\
& \quad+\int_{Z}\left\|D y_{1}\right\|^{p-2}\left(D y_{1}, D \psi\right)_{\mathbb{R}^{\mathbb{N}}} \xi_{\varepsilon}\left(\left(y_{1}-y_{2}\right)^{-}\right) d z \\
& \quad+\int_{Z}\left\|D y_{2}\right\|^{p-2}\left(D y_{2}, D \psi\right)_{\mathbb{R}^{\mathbb{N}}}\left(\varepsilon-\xi_{\varepsilon}\left(\left(y_{1}-y_{2}\right)^{-}\right)\right) d z \\
& \leq \int_{Z}\left\|D y_{1}\right\|^{p-2}\left(D y_{1}, D \psi\right)_{\mathbb{R}^{\mathbb{N}} \xi_{\varepsilon}}\left(\left(y_{1}-y_{2}\right)^{-}\right) d z \\
& \quad+\int_{Z}\left\|D y_{2}\right\|^{p-2}\left(D y_{2}, D \psi\right)_{\mathbb{R}^{\mathbb{N}}}\left(\varepsilon-\xi_{\varepsilon}\left(\left(y_{1}-y_{2}\right)^{-}\right)\right) d z .
\end{aligned}
$$

Returning to (4.5), using (4.7), and dividing by $\varepsilon>0$, we get

$$
\begin{aligned}
\int_{Z}\left\|D y_{1}\right\|^{p-2}\left(D y_{1}, D \psi\right)_{\mathbb{R}^{\mathbb{N}}} \frac{1}{\varepsilon} \xi_{\varepsilon}\left(\left(y_{1}-y_{2}\right)^{-}\right) d z \\
\quad+\int_{Z}\left\|D y_{2}\right\|^{p-2}\left(D y_{2}, D \psi\right)\left(1-\frac{1}{\varepsilon} \xi_{\varepsilon}\left(\left(y_{1}-y_{2}\right)^{-}\right)\right) d z \\
\geq\left\langle u, \frac{1}{\varepsilon} \xi_{\varepsilon}\left(\left(y_{1}-y_{2}\right)^{-}\right) \psi\right\rangle+\left\langle u_{2},\left(1-\frac{1}{\varepsilon} \xi_{\varepsilon}\left(\left(y_{1}-y_{2}\right)^{-}\right)\right) \psi\right\rangle .
\end{aligned}
$$


We observe that

$$
\begin{gathered}
\frac{1}{\varepsilon} \xi_{\varepsilon}\left(\left(y_{1}-y_{2}\right)^{-}(z)\right) \longrightarrow X\left\{y_{1}<y_{2}\right\} \\
X\left\{y_{1} \geq y_{2}\right\} \\
=1-X\left\{y_{1}<y_{2}\right\}
\end{gathered}
$$

Therefore, if we pass to the limit as $\varepsilon \downarrow 0$ in (4.8), we obtain

$$
\begin{aligned}
& \int_{\left\{y_{1}<y_{2}\right\}}\left\|D y_{1}\right\|^{p-2}\left(D y_{1}, D \psi\right)_{\mathbb{R}^{\mathbb{N}}} d z+\int_{\left\{y_{1} \geq y_{1}\right\}}\left\|D y_{2}\right\|^{p-2}\left(D y_{2}, D \psi\right)_{\mathbb{R}^{\mathbb{N}}} d z \\
& \geq \int_{\left\{y_{1}<y_{2}\right\}} u_{1} \psi d z+\int_{\left\{y_{1} \geq y_{2}\right\}} u_{2} \psi d z .
\end{aligned}
$$

Since $y=\min \left\{y_{1}, y_{2}\right\} \in W^{1, p}(Z)$, we have

$$
D y(z)= \begin{cases}D y_{1}(z) & \text { for a.a. } z \in\left\{y_{1}<y_{2}\right\} \\ D y_{2}(z) & \text { for a.a. } z \in\left\{y_{1} \geq y_{2}\right\}\end{cases}
$$

Therefore

Also if $u=X_{\left\{y_{1}<y_{2}\right\}} u_{1}+X_{\left\{y_{1} \geq y_{2}\right\}} u_{2}$, then $u \in L^{p^{\prime}}(Z)$ and $u(z) \in \partial j(z, y(z))$ a.e. on $Z$.

$$
\int_{Z}\|D y\|^{p-2}(D y, D \psi)_{\mathbb{R}^{\mathbb{N}}} d z \geq \int_{Z} u \psi d z
$$

for some $u \in L^{p^{\prime}}(Z)$ with $u(z) \in \partial j(z, y(z))$ a.e. on $Z$. Since $\psi \in C_{c}^{1}(Z)_{+}$was arbitrary and $C_{c}^{1}(Z)_{+}$is dense in $W_{0}^{1, p}(Z)_{+}$, from (4.12) we conclude that $y=\min \left\{y_{1}, y_{2}\right\} \in W^{1, p}(Z)$ is an upper solution for problem (1.1).

Arguing similarly, we can also show that the set of lower solutions for problem (1.1) is upward directed. Namely, we have the following.

Lemma 4.2. If $v_{1}, v_{2} \in W^{1, p}(Z)$ are lower solutions for problem (1.1) and $v=\max \left\{v_{1}, v_{2}\right\} \in$ $W^{1, p}(Z)$, then $v$ is also a lower solution for problem (1.1).

Now that we have established that the sets of upper solutions and of lower solutions are directed, we will show that problem (1.1) admits the smallest positive solution and the largest negative solution. To this end, we need to strengthen the hypotheses on $j(z, x)$.

$H(j)_{2}: j: Z \times \mathbb{R} \rightarrow \mathbb{R}$ is a function such that $j(z, 0)=0$ a.e. on $Z, \partial j(z, 0)=\{0\}$ a.e. on $Z$; it satisfies hypotheses $H(j)_{1}(\mathrm{i}) \rightarrow(\mathrm{iv})$ and (vi) and

(v) there exists $\hat{\eta} \in L^{\infty}(Z)_{+}$such that

$$
\lambda_{1}<\liminf _{x \rightarrow 0} \frac{u}{|x|^{p-2} x} \leq \limsup _{x \rightarrow 0} \frac{u}{|x|^{p-2} x} \leq \widehat{\eta}(z)
$$

uniformly for almost all $z \in Z$ and all $u \in \partial j(z, x)$. 
Remark 4.3. Note that in the new hypotheses, we have strengthened the condition concerning the behavior of $\partial j(z, \cdot)$ near the origin. This has as a consequence that we can replace the origin as a lower solution in the positive axis and as an upper solution in the negative axis (see Section 3), by functions which are strictly positive and strictly negative, respectively. This is done in the next lemma.

Lemma 4.4. If hypotheses $H(j)_{2}$ hold, then problem (1.1) has a strict lower solution $\underline{x} \in \operatorname{int} C_{0}^{1}(\bar{Z})_{+}$ and a strict upper solution $\bar{v} \in-\operatorname{int} C_{0}^{1}(\bar{Z})_{+}$.

Proof. By virtue of hypothesis $H(j)_{2}(\mathrm{v})$, we can find $\widehat{c}>\lambda_{1}$ and $\delta>0$ such that for almost all $z \in Z$, all $x \in[0, \delta]$, and all $u \in \partial j(z, x)$, we have

$$
\widehat{c} x^{p-1} \leq u
$$

We know that for $u_{1}$ the principal eigenfunction of $\left(-\Delta_{p}, W_{0}^{1, p}(Z)\right)$ (i.e., $\left.m \equiv 1\right)$, we have that $u_{1} \in \operatorname{int} C_{0}^{1}(\bar{Z})_{+}$. Thus we can find $\mu \in(0,1)$ small enough such that $0<\mu u_{1}(z) \leq \delta$ for all $z \in Z$. Let $\bar{x} \in \operatorname{int} C_{0}^{1}(\bar{Z})_{+}$be the strict upper solution for problem (1.1) obtained in Proposition 3.4. Invoking Lemma 3.5, we can find $t>1$ such that $t \bar{x}-\mu u_{1} \in \operatorname{int} C_{0}^{1}(\bar{Z})_{+}$. We set

$$
\underline{x}=\frac{\mu}{t} u_{1} \in \operatorname{int} C_{0}^{1}(\bar{Z})_{+} .
$$

Note that since $t>1, \underline{x}(z) \in(0, \delta]$ for all $z \in Z$. Hence

$$
\begin{aligned}
-\operatorname{div}\left(\|D \underline{x}(z)\|^{p-2}\right) D \underline{x}(z) & =\lambda_{1}|\underline{x}(z)|^{p-2} \underline{x}(z) \\
& <\widehat{c}|\underline{x}(z)|^{p-2} \underline{x}(z) \\
& \leq u(z) \text { a.e. on } Z
\end{aligned}
$$

for every $u \in L^{p^{\prime}}(Z)$ with $u(z) \in \partial j(z, \underline{x}(z))$ a.e. on $Z$ (see (4.14)). Hence for all $\psi \in W^{1, p}(Z)_{+}$, we have

$$
\int_{Z}\|D \underline{x}\|^{p-2}(D \underline{x}, D \psi)_{\mathbb{R}^{\mathbb{N}}} d z<\int_{Z} u \psi d z
$$

$\Longrightarrow \underline{x} \in \operatorname{int} C_{0}^{1}(\bar{Z})_{+}$is a strict lower solution for problem (1.1).

Note that from the definition of $\underline{x}$, we have $\bar{x}-\underline{x} \in \operatorname{int} C_{0}^{1}(\bar{Z})_{+}$.

A similar reasoning applied on the negative semiaxis produces an upper solution $\bar{v}=$ $(\bar{\mu} / t)\left(-u_{1}\right)$ for some $0 \leq \bar{\mu}<1<\bar{t}$. Then $\bar{v} \in-\operatorname{int} C_{0}^{1}(\bar{Z})_{+}$and as for $\underline{x}$, we will have $\bar{v}-\underline{v} \in$ $\operatorname{int} C_{0}^{1}(\bar{Z})_{+}$.

Using $\{\underline{x}, \bar{x}\}$ and $\{\underline{v}, \bar{v}\}$, we introduce the following order intervals in $W_{0}^{1, p}(Z)$ :

$$
\begin{aligned}
& {[\underline{x}, \bar{x}]=\left\{x \in W_{0}^{1, p}(Z): \underline{x}(z) \leq x(z) \leq \bar{x}(z) \text { a.e. on } Z\right\}} \\
& {[\underline{v}, \bar{v}]=\left\{v \in W_{0}^{1, p}(Z): \underline{v}(z) \leq v(z) \leq \bar{v}(z) \text { a.e. on } Z\right\}}
\end{aligned}
$$


In the next proposition, we establish the existence of the smallest solution of (1.1) in $[\underline{x}, \bar{x}]$ and of the greatest solution of $(1.1)$ in $[\underline{v}, \bar{v}]$.

Proposition 4.5. If hypotheses $H(j)_{2}$ hold, then problem (1.1) admits the smallest solution in the order interval $[\underline{x}, \bar{x}]$ and the greatest solution in the order interval $[\underline{v}, \bar{v}]$.

Proof. We will show that the existence of the smallest solution in $[\underline{x}, \bar{x}]$ and the proof of the greatest solution in $[\underline{v}, \bar{v}]$ is similar.

Let $S_{+}$be the set of solutions of (1.1) belonging in the order interval $E_{+}=[\underline{x}, \bar{x}]$. We will show that $S_{+}$is downward directed. So let $x_{1}, x_{2} \in S_{+}$. In particular both $x_{1}, x_{2}$ are upper solutions for problem (1.1). Then Lemma 4.1 implies that $\hat{x}=\min \left\{x_{1}, x_{2}\right\} \in W_{0}^{1, p}(Z)$ is also an upper solution for problem (1.1). We set

$$
\widehat{E}_{+}=[\underline{x}, \widehat{x}]=\left\{x \in W_{0}^{1, p}(Z): \underline{x}(z) \leq x(z) \leq \widehat{x}(z)\right\} .
$$

Using standard truncation and penalization techniques, we can obtain $\widehat{x}_{0} \in \widehat{E}_{+}$ a solution of (1.1) (see Carl-Heikkilä [33] and Gasiński -Papageorgiou [19]). Nonlinear regularity theory implies that $\widehat{x}_{0} \in C_{0}^{1}(\bar{Z})_{+}$and we have

$$
\begin{aligned}
& \underline{x} \leq \widehat{x}_{0} \leq \min \left\{x_{1}, x_{2}\right\} \\
& \Longrightarrow S_{+} \text {is downward directed. }
\end{aligned}
$$

Consider a chain $\Gamma \subseteq S_{+}$(i.e., a totally ordered subset of $S_{+}$). By virtue of $[34$, Corollary 7, page 336] by Dunford-Schwartz, we can find $\left\{x_{n}\right\}_{n \geq 1} \subseteq \Gamma$ such that

$$
\inf _{n \geq 1} x_{n}=\inf \Gamma
$$

Because of (4.20), we may assume that $\left\{x_{n}\right\}_{n \geq 1}$ is decreasing. Also since the $x_{n}$ 's are solutions of (1.1) in $E_{+}$, we see that there exists $c_{3}>0$ such that

$$
\left\|D x_{n}\right\|_{p} \leq c_{3} \quad \forall n \geq 1 .
$$

Therefore $\left\{x_{n}\right\}_{n \geq 1} \subseteq W_{0}^{1, p}(Z)$ is bounded and so we may assume that

$$
x_{n} \stackrel{w}{\longrightarrow} \hat{y} \text { in } W_{0}^{1, p}(Z), \quad x_{n} \longrightarrow \widehat{y} \text { in } L^{p}(Z) \quad \text { as } n \longrightarrow \infty
$$

We can find $u_{n} \in L^{p^{\prime}}(Z)$ with $u_{n}(z) \in \partial j\left(z, x_{n}(z)\right)$ a.e. on $Z$ such that

$$
A\left(x_{n}\right)=u_{n} \quad \forall n \geq 1 .
$$

Because of hypothesis $H(j)_{2}(\mathrm{iii}),\left\{u_{n}\right\}_{n \geq 1} \subseteq L^{p^{\prime}}(Z)$ is bounded. So we may assume that $u_{n} \stackrel{w}{\rightarrow} \widehat{u}$ in $L^{p^{\prime}}(Z)$ as $n \rightarrow \infty$. By virtue of Hu-Papageorgiou [35, Proposition 3.10, page 694], 
we have $\widehat{u}(z) \in \partial j(z, \widehat{y}(z))$ a.e. on $Z$ (recall that the multifunction $x \rightarrow \partial j(z, x)$ has closed graph; see Clarke [1, page 29]). From (4.24), we have

$$
\left\langle A\left(x_{n}\right), x_{n}-\widehat{y}\right\rangle=\int_{Z} u_{n}\left(x_{n}-\widehat{y}\right) d z \longrightarrow 0 \quad \text { as } n \longrightarrow \infty
$$

Since $A$ is maximal monotone, we have (see Gasiński -Papageorgiou [19, page 84])

$$
\begin{aligned}
& \left\langle A\left(x_{n}\right), x_{n}\right\rangle \longrightarrow\langle A(\widehat{y}), \hat{y}\rangle, \\
& \Longrightarrow\left\|D x_{n}\right\|_{p} \longrightarrow\|D \hat{y}\|_{p} .
\end{aligned}
$$

Recalling that $D x_{n} \stackrel{w}{\rightarrow} D \widehat{y}$ in $L^{p}\left(Z, \mathbb{R}^{\mathbb{N}}\right)$, we infer that $D x_{n} \rightarrow D \widehat{y}$ in $L^{p}\left(Z, \mathbb{R}^{\mathbb{N}}\right)$ (KadecKlee property) and so we conclude that $x_{n} \rightarrow \widehat{y}$ in $W_{0}^{1, p}(Z)$ as $n \rightarrow \infty$. So, if we pass to the limit as $n \rightarrow \infty$ in (4.24) and since $A$ is demicontinuous, it follows that

$$
A(\widehat{y})=\widehat{u}
$$

with $\widehat{u} \in L^{p^{\prime}}(Z), \widehat{u}(z) \in \partial j(z, \widehat{y}(z))$ a.e. on $Z$. Therefore $\widehat{y} \in S_{+}$and $\widehat{y}=\inf \Gamma$. Because $\Gamma$ was an arbitrary chain, invoking Zorn's lemma, we obtain $x_{*} \in S_{+}$a minimal element. Then from (4.20), we conclude that $x_{*}$ is the smallest solution of (1.1) in $E_{+}$.

We will use this proposition to produce the smallest positive and the greatest negative solutions for problem (1.1).

Proposition 4.6. If hypotheses $H(j)_{2}$ hold, then problem (1.1) has the smallest nontrivial solution $y_{+} \in \operatorname{int} C_{0}^{1}(\bar{Z})_{+}$and the greatest nontrivial negative solution $y_{-} \in-\operatorname{int} C_{0}^{1}(\bar{Z})_{+}$.

Proof. Let $\underline{x}_{n}=\varepsilon_{n} u_{1}$ with $\varepsilon_{n} \downarrow 0$ and let $E_{+}^{n}=\left[\underline{x}_{n}, \bar{x}\right]$. From Proposition 4.5, we know that problem (1.1) admits the smallest solution $x_{*}^{n}$ in the order interval $E_{+}^{n}$. We know that $\left\{x_{*}^{n}\right\}_{n \geq 1} \subseteq$ $W_{0}^{1, p}(Z)$ is bounded and so we may assume that

$$
x_{*}^{n} \stackrel{w}{\longrightarrow} y_{+} \text {in } W_{0}^{1, p}(Z), \quad x_{*}^{n} \longrightarrow y_{+} \text {in } L^{p}(Z) \quad \text { as } n \longrightarrow \infty
$$

We know that

$$
A\left(x_{*}^{n}\right)=u_{*}^{n} \quad \forall n \geq 1,
$$

with $u_{*}^{n} \in L^{p^{\prime}}(Z), u_{*}^{n}(z) \in \partial j\left(z, x_{*}^{n}(z)\right)$ a.e. on $Z$. Taking duality brackets with $x_{*}^{n}-y_{+}$and arguing as before, we can check that

$$
x_{*}^{n} \longrightarrow y_{+} \text {in } W_{0}^{1, p}(Z) \text { as } n \longrightarrow \infty .
$$


Suppose that $y_{+}=0$. Then $\left\|x_{*}^{n}\right\| \rightarrow 0$ as $n \rightarrow \infty$. We set $w_{n}=x_{*}^{n} /\left\|x_{*}^{n}\right\|, n \geq 1$. Then by passing to a suitable subsequence if necessary, we may assume that

$$
w_{n} \stackrel{w}{\longrightarrow} w \text { in } W_{0}^{1, p}(Z), \quad w_{n} \longrightarrow w \text { in } L^{p}(Z) n \longrightarrow \infty
$$

From (4.29), we have

$$
A\left(w_{n}\right)=\frac{u_{*}^{n}}{\left\|x_{*}^{n}\right\|^{p-1}} \quad \forall n \geq 1
$$

and so taking duality brackets with $w_{n}-w$, again we obtain

$$
\lim _{n \rightarrow \infty}\left\langle A\left(w_{n}\right), w_{n}-w\right\rangle=0
$$

from which it follows that

$$
w_{n} \longrightarrow w \text { in } W_{0}^{1, p}(Z)
$$

and so we have $\|w\|=1, w \neq 0$. By virtue of hypothesis $H(j)_{2}(\mathrm{v})$, we can find $\delta>0$ such that for almost all $z \in Z$, all $0<|x| \leq \delta$, and all $u \in \partial j(z, x)$, we have

$$
\beta \leq \frac{u}{|x|^{p-2} x} \leq \widehat{\eta}(z)+1 \quad \text { with } \beta>\lambda_{1}
$$

In addition, due to hypothesis $H(j)_{2}$ (iii), for almost all $z \in Z$, all $|x| \geq \delta$, and all $u \in \partial j(z, x)$, we have

$$
|u| \leq \alpha(z)+c|x|^{p-1} \leq\left(\frac{\alpha(z)}{\delta^{p-1}}+c\right)|x|^{p-1}
$$

So finally from (4.35) and (4.36), we infer that

$$
|u| \leq c_{3}|x|^{p-1} \text { for a.a. } z \in Z \text {, all } x \in \mathbb{R} \text {, and all } u \in \partial j(z, x) .
$$

From (4.37) it follows that

$$
\left\{\frac{u_{*}^{n}}{\left\|x_{*}^{n}\right\|^{p-1}}\right\}_{n \geq 1} \subseteq L^{p^{\prime}}(Z) \text { is bounded }
$$

Therefore we may assume that

$$
h_{n}=\frac{u_{*}^{n}}{\left\|x_{*}^{n}\right\|^{p-1}} \stackrel{w}{\longrightarrow} h \text { in } L^{p^{\prime}}(Z) .
$$


For $\varepsilon>0$ and $n \geq 1$ we introduce the sets

$$
\begin{aligned}
& \Sigma_{+}^{n}=\left\{z \in Z: x_{n}(z)>0, \beta-\varepsilon \leq \frac{u_{*}^{n}(z)}{x_{*}^{n}(z)^{p-1}} \leq \widehat{\eta}(z)+\varepsilon\right\}, \\
& \Sigma_{-}^{n}=\left\{z \in Z: x_{n}(z)<0, \beta-\varepsilon \leq \frac{u_{*}^{n}(z)}{x_{*}^{n}(z)^{p-1}} \leq \widehat{\eta}(z)+\varepsilon\right\} .
\end{aligned}
$$

Since $x_{*}^{n} \rightarrow 0$ in $W_{0}^{1, p}(Z)$, we may assume (at least for a subsequence) that $x_{*}^{n}(z) \rightarrow 0$ a.e. on $Z$. Then $x_{*}^{n}(z) \rightarrow 0^{+}$a.e. on $\{w>0\}$ and $x_{*}^{n}(z) \rightarrow 0^{-}$a.e. on $\{w<0\}$ and so because of hypothesis $H(j)_{2}(\mathrm{v})$, we have

$$
X_{\Sigma_{+}^{n}}(z) \longrightarrow 1 \text { a.e. on }\{w>0\}, \quad X_{\Sigma_{-}^{n}}(z) \longrightarrow 1 \text { a.e. on }\{w<0\}
$$

Then we have

$$
X \Sigma_{+}^{n} \frac{u_{*}^{n}}{\left\|x_{*}^{n}\right\|^{p-1}} \stackrel{w}{\longrightarrow} h \quad \text { in } L^{p^{\prime}}(\{w>0\}), \quad X \Sigma_{-}^{n} \frac{u_{*}^{n}}{\left\|x_{*}^{n}\right\|^{p-1}} \stackrel{w}{\longrightarrow} h \quad \text { in } L^{p^{\prime}}(\{w<0\}) .
$$

From the definition of the set $\Sigma_{+}^{n}$, we have

$$
\begin{aligned}
\chi_{\Sigma_{+}^{n}}(z)(\beta-\varepsilon) w_{n}(z)^{p-1} & \leq \chi_{\Sigma_{+}^{n}}(z) \frac{u_{*}^{n}(z)}{\chi_{*}^{n}(z)^{p-1}} w_{n}(z)^{p-1}=\chi_{\Sigma_{+}^{n}}(z) h_{n}(z) \\
& \leq \chi_{\Sigma_{+}^{n}}(z)(\widehat{\eta}(z)+\varepsilon) w_{n}(z)^{p-1} \quad \text { a.e. on } Z .
\end{aligned}
$$
we obtain

Taking weak limits in $L^{p^{\prime}}(\{w>0\})$, via Mazur's lemma, and since $\varepsilon>0$ was arbitrary,

$$
\beta w(z)^{p-1} \leq h(z) \leq \widehat{\eta}(z) w(z)^{p-1} \text { a.e. on }\{w>0\} .
$$

Similarly working on $\Sigma_{-}^{n}$, we obtain

$$
\widehat{\eta}(z)|w(z)|^{p-2} w(z) \leq h(z) \leq \beta|w(z)|^{p-2} w(z) \text { a.e. on }\{w<0\} .
$$

Moreover, from (4.36) we see that

$$
h(z)=0 \quad \text { a.e. on }\{w=0\} .
$$

So from (4.44), (4.45), and (4.46) it follows that

$$
h(z)=\xi_{1}(z)|w(z)|^{p-2} w(z) \quad \text { a.e. on } Z \text {, }
$$


with $\xi_{1} \in L^{\infty}(Z)_{+}$and $\lambda_{1}<\xi_{1}(z) \leq \widehat{\eta}(z)$ a.e. on $Z$. Therefore, if we pass to the limit as $n \rightarrow \infty$ in (4.32), we obtain

$$
\begin{aligned}
& A(w)=\xi_{1}|w|^{p-2} w \\
& \Longrightarrow\left\{\begin{array}{c}
-\operatorname{div}\left(\|D w(z)\|^{p-2} D w(z)\right)=\xi_{1}(z)|w(z)|^{p-2} w(z) \text { a.e. on } Z, \\
\left.w\right|_{\partial Z}=0 \quad w \neq 0
\end{array}\right\} .
\end{aligned}
$$

Note that $\hat{\lambda}_{1}\left(\xi_{1}\right)<\hat{\lambda}\left(\lambda_{1}\right)=1$. So from (4.48), it follows that $w$ must change sign. But $w_{n}=x_{*}^{n} /\left\|x_{*}^{n}\right\| \geq 0$ for all $n \geq 1$ and so $w \geq 0$, a contradiction. This proves that we cannot have $y_{+}=0$, hence $y_{+} \neq 0$ and of course $y_{+} \geq 0$. Moreover, as before we can check that

$$
x_{*}^{n} \longrightarrow y_{+} \quad \text { in } W_{0}^{1, p}(Z), \quad A\left(y_{+}\right)=u_{+} \quad(\text { see }(4.29)),
$$

with $u_{+} \in L^{p^{\prime}}(Z), u_{+}(z) \in \partial j\left(z, y_{+}(z)\right)$ a.e. on Z. It follows that

$$
\begin{gathered}
-\operatorname{div}\left(\left\|D y_{+}(z)\right\|^{p-2} D y_{+}(z)\right)=u_{+}(z) \quad \text { a.e. on } Z, \\
\left.y_{+}\right|_{\partial z}=0 .
\end{gathered}
$$

From nonlinear regularity theory we have $y_{+} \in C_{0}^{1}(\bar{Z})_{+}, y_{+} \neq 0$. Moreover, from hypothesis $H(j)_{2}(\mathrm{v})$ (the sign condition), we have $u_{+}(z) \geq 0$ a.e. on $Z$. So via the nonlinear strong maximum principle of Vázquez [23], we obtain that $y_{+} \in \operatorname{int} C_{0}^{1}(\bar{Z})_{+}$.

We claim that $y_{+}$is the smallest nontrivial positive solution of (1.1). Indeed let $\hat{y}$ be another nontrivial positive solution of (1.1) and assume that $\hat{y} \leq \bar{x}$. As above we can verify that $\widehat{y} \in \operatorname{int} C_{0}^{1}(\bar{Z})_{+}$. Using Lemma 3.5, we can find $\widehat{\varepsilon}>0$ such that $\widehat{\varepsilon} u_{1} \leq \widehat{y}$. Then for $n \geq 1$ large we have $\varepsilon_{n} u_{1} \leq \widehat{\varepsilon} u_{1} \leq \hat{y} \leq \bar{x}$. So for $n \geq 1$ large, working on the order interval $\left[\varepsilon_{n} u_{1}, \hat{y}\right]$, we can obtain a solution $y_{0}$ of (1.1) in the interval. Then $x_{*}^{n} \leq y_{0}$ for $n \geq 1$ large and so $y_{+} \leq y_{0} \leq \hat{y}$. This proves the claim.

In a similar fashion working on $E_{-}^{n}=\left[\underline{v}, \bar{v}_{n}\right]$ with $\bar{v}_{n}=\varepsilon_{n}\left(-u_{1}\right)$, we obtain $y_{-} \in$ $\operatorname{int} C_{0}^{1}(\bar{Z})_{+}$, the largest nontrivial negative solution of (1.1).

Now we are ready to conclude our plan and produce a nontrivial nodal solution. More precisely, using variational methods and Theorem 2.5, we will obtain a nontrivial solution $y_{0}$ of (1.1) in the order interval $\left[y_{-}, y_{+}\right]$distinct from $y_{-}$and $y_{+}$. Evidently $y_{0}$ must be a sign changing (nodal) solution. We will achieve this by exploiting the variational characterization of $\lambda_{2}$ provided in (2.10). This requires a further strengthening of hypotheses $H(j)_{1}$. Suppose $f: Z \times \mathbb{R} \rightarrow \mathbb{R}$ is a measurable function with the following property, for every $r>0$ there exists $\alpha_{r} \in L^{\infty}(Z)_{+}$such that $|f(z, x)| \leq \alpha_{r}(z)$ for a.a. $z \in Z$ and all $|x| \leq r$. Then we define

$$
f_{1}(z, x)=\liminf _{x^{\prime} \rightarrow x} f(z, x), \quad f_{2}(z, x)=\liminf _{x^{\prime} \rightarrow x} f(z, x)
$$


which for almost all $z \in Z$ are finite. We assume that $f_{1}$ and $f_{2}$ are supmeasurable, namely, for every $x: Z \rightarrow \mathbb{R}$ measurable function the functions $z \rightarrow f_{1}(z, x(z))$ and $z \rightarrow f_{2}(z, x(z))$ are both measurable. We set

$$
j(z, x)=\int_{0}^{x} f(z, r) d r \quad \forall(z, x) \in Z \times \mathbb{R}
$$

Evidently $(z, x) \rightarrow j(z, x)$ is measurable and for almost all $z \in Z, x \rightarrow j(z, x)$ is locally Lipschitz and its generalized subdifferential satisfies

$$
\partial j(z, x) \subseteq\left[f_{1}(z, x), f_{2}(z, x)\right] \quad \text { (see Chang [27]). }
$$

Clearly $j(z, 0)=0$ a.e. on $Z$ and if for almost all $z \in Z, f(z, \cdot)$ is continuous at $x=0$, then $\partial j(z, 0)=\{0\}$ a.e. on $Z$. Then the new hypotheses on the nonsmooth potential $j(z, x)$ are the following:

$$
\begin{aligned}
& H(j)_{3}: j: Z \times \mathbb{R} \rightarrow \mathbb{R} \text { is a function such that } j(z, x)=\int_{0}^{x} f(z, r) d r \text { with } f: Z \times \mathbb{R} \rightarrow \mathbb{R} \\
& \text { satisfying } \\
& \text { (i) }(z, x) \rightarrow f(z, x) \text { is measurable with } f_{1} \text { and } f_{2} \text { supmeasurable; } \\
& \text { (ii) for almost all } z \in Z, f(z, \cdot) \text { is continuous at } x=0 \text {; } \\
& \text { (iii) }|f(z, x)| \leq \alpha(z)+c|x|^{p-1} \text { for a.a. } z \in Z \text {, all } x \in \mathbb{R} \text {, with } \alpha \in L^{\infty}(Z)_{+}, c>0 \text {; } \\
& \text { (iv) there exists } \theta \in L^{\infty}(Z)_{+} \text {satisfying } \theta(z) \leq \lambda_{1} \text { a.e. on } Z \text { with strict inequality on } \\
& \text { a set of positive measure, such that }
\end{aligned}
$$

$$
\limsup _{|x| \rightarrow \infty} \frac{f_{2}(z, x)}{|x|^{p-2} x} \leq \theta(z) \quad \text { uniformly for a.a. } z \in Z \text {; }
$$

(v) there exists $\hat{\eta} \in L^{\infty}(Z)_{+}$such that

$$
\lambda_{2}<\liminf _{x \rightarrow 0} \frac{f_{1}(z, x)}{|x|^{p-2} x} \leq \limsup _{x \rightarrow 0} \frac{f_{2}(z, x)}{|x|^{p-2} x} \leq \widehat{\eta}(z) \quad \text { uniformly for a.a. } z \in Z
$$

(vi) for almost all $z \in Z$ and all $x \in \mathbb{R}$, we have $f_{1}(z, x) x \geq 0$ (sign condition).

Theorem 4.7. If hypotheses $H(j)_{3}$ hold, then problem (1.1) has at least three nontrivial solutions $x_{0}, v_{0}, y_{0}$ such that $x_{0} \in \operatorname{int} C_{0}^{1}(\bar{Z})_{+}, v_{0} \in-\operatorname{int} C_{0}^{1}(\bar{Z})_{+}$, and $y_{0} \in C_{0}^{1}(\bar{Z})$ a (nodal) solution.

Proof. From Theorem 3.8, we have the two constant sign solutions $x_{0} \in \operatorname{int} C_{0}^{1}(\bar{Z})_{+}$and $v_{0} \in-\operatorname{int} C_{0}^{1}(\bar{Z})_{+}$. Let $y_{+} \in \operatorname{int} C_{0}^{1}(\bar{Z})_{+}$and $y_{-} \in-\operatorname{int} C_{0}^{1}(\bar{Z})_{+}$be the two extremal constant sign solutions from Proposition 4.6. We have

$$
A\left(y_{ \pm}\right)=u_{ \pm} \quad \text { with } u_{ \pm} \in L^{p^{\prime}}(Z), \quad u_{ \pm}(z) \in \partial j\left(z, u_{ \pm}(z)\right) \quad \text { a.e. on } Z \text {. }
$$


We introduce the following functions

$$
\begin{aligned}
& \widehat{f}_{+}(z, x)= \begin{cases}0 & \text { if } x<0, \\
f(z, x) & \text { if } 0 \leq x \leq y_{+}(z), \\
u_{+}(z) & \text { if } y_{+}(z)<x,\end{cases} \\
& \widehat{f}_{-}(z, x)= \begin{cases}u_{-}(z) & \text { if } x<y_{-}(z), \\
f(z, x) & \text { if } y_{-}(z) \leq x \leq 0, \\
0 & \text { if } 0<x,\end{cases} \\
& \widehat{f}(z, x)= \begin{cases}u_{-}(z) & \text { if } x<y_{-}(z), \\
f(z, x) & \text { if } y_{-}(z) \leq x \leq y_{+}(z), \\
u_{+}(z) & \text { if } y_{+}(z)<x .\end{cases}
\end{aligned}
$$

We consider the corresponding potential functions defined by

$$
\widehat{j}_{+}(z, x)=\int_{0}^{x} \widehat{f}_{+}(z, r) d r, \quad \hat{j}_{-}(z, x)=\int_{0}^{x} \widehat{f}_{-}(z, r) d r, \quad \widehat{j}(z, x)=\int_{0}^{x} \widehat{f}(z, r) d r .
$$

Then we define the following locally Lipschitz Euler functionals on $W_{0}^{1, p}(Z)$ :

$$
\begin{aligned}
\varphi_{0,+}(x) & =\frac{1}{p}\|D x\|_{p}^{p}-\int_{Z} \widehat{j}_{+}(z, x(z)) d z, \\
\varphi_{0,-}(x) & =\frac{1}{p}\|D x\|_{p}^{p}-\int_{Z} \widehat{j}_{-}(z, x(z)) d z, \\
\varphi_{0}(x) & =\frac{1}{p}\|D x\|_{p}^{p}-\int_{Z} \hat{j}(z, x(z)) d z .
\end{aligned}
$$

We will use the following order intervals in $W_{0}^{1, p}(Z)$ :

$$
I_{+}=\left[0, y_{+}\right], \quad I_{-}=\left[y_{-}, 0\right], \quad I=\left[y_{-}, y_{+}\right] .
$$

The critical points of $\varphi_{0,+}$ are in $I_{+}$, the critical points of $\varphi_{0,-}$ are in $I_{-}$, and the critical points of $\varphi_{0}$ are in $I$. We show this for $\varphi_{0,+} ;$ the proof for the rest is similar. 
So let $x \in W_{0}^{1, p}(Z)$ be a critical point of $\varphi_{0,+}$. Then we have $0 \in \partial \varphi_{0,+}(x)$ and so $A(x)=u$ with $u \in L^{p^{\prime}}(Z), u(z) \in \partial \widehat{j}_{+}(z, x(z))$ a.e. on $Z$. If we act with the test function $\left(x-y_{+}\right)^{+} \in$ $W_{0}^{1, p}(Z)$ we obtain

$$
\begin{aligned}
\left\langle A(x),\left(x-y_{+}\right)^{+}\right\rangle & =\int_{Z} u\left(x-y_{+}\right)^{+} d z \\
& =\int_{\left\{x>y_{+}\right\}} u\left(x-y_{+}\right) d z \\
& =\int_{Z} u_{+}\left(x-y_{+}\right)^{+} d z \\
& =\left\langle A\left(y_{+}\right),\left(x-y_{+}\right)^{+}\right\rangle, \\
& \Longrightarrow\left\langle A(x)-A\left(y_{+}\right),\left(x-y_{+}\right)^{+}\right\rangle \\
& =\int_{\left\{x>y_{+}\right\}}\left(\|D x\|^{p-2} D x-\left\|D y_{+}\right\|^{p-2} D y_{+}, D x-D y_{+}\right)_{\mathbb{R}^{\mathbb{N}}} d z=0, \\
& \Longrightarrow\left|\left\{x>y_{+}\right\}\right|_{N}=0 \quad\left(|\cdot|_{N} \text { being the Lebesgue measure on } \mathbb{R}^{\mathbb{N}}\right), \text { i.e., } x \leq y_{+} .
\end{aligned}
$$

Similarly we show that $0 \leq x$. Hence $x \in I_{+}$.

Since the critical points of $\varphi_{0,+}$ are in $I_{+}$, it follows that $\left\{0, y_{+}\right\}$are the only critical points of $\varphi_{0,+}$. From hypothesis $H(j)_{3}(v)$, we can find $\delta>0$ small such that

$$
\lambda_{2} x^{p-1}<u \text { for a.a. } z \in Z \text { and all } x \in[0, \delta] \text { and all } u \in \partial j(z, x)
$$

We choose $\varepsilon>0$ small such that

$$
\varepsilon u_{1}(z) \leq \min \left\{y_{+}(z), \delta\right\} \quad \forall z \in \bar{Z}
$$

We know that

$$
\begin{array}{r}
j(z, x)=\int_{0}^{x} f(z, r) d r \quad \text { for a.a. } z \in Z, \text { all } x \in \mathbb{R}, \\
f(z, r) \in \partial j(z, r) \quad \text { for a.a. } z \in Z, \text { a.a. } r \in \mathbb{R} .
\end{array}
$$

Then from (4.62), (4.63), and (4.64), we have

$$
\widehat{j}_{+}\left(z, \varepsilon u_{1}(z)\right)=\widehat{j}\left(z, \varepsilon u_{1}(z)\right)=\int_{0}^{\varepsilon u_{1}(z)} f(z, r) d r>\frac{\lambda_{2}}{p} \varepsilon^{p} u_{1}(z)^{p} \quad \text { a.e. on } Z \text {. }
$$


Hence

$$
\begin{aligned}
\varphi_{0,+}\left(\varepsilon u_{1}\right) & =\frac{\varepsilon^{p}}{p}\left\|D u_{1}\right\|_{p}^{p}-\int_{Z} \widehat{j}_{+}\left(z, \varepsilon u_{1}(z)\right) d z \\
& <\frac{\varepsilon^{p}}{p}\left(\left\|D u_{1}\right\|_{p}^{p}-\lambda_{2}\left\|u_{1}\right\|_{p}^{p}\right)(\text { see }(4.65)) \\
& <\frac{\varepsilon^{p}}{p}\left(\left\|D u_{1}\right\|_{p}^{p}-\lambda_{1}\left\|u_{1}\right\|_{p}^{p}\right)=0(\text { see }(2.6) \text { with } m \equiv 1), \\
\Longrightarrow \inf _{W_{0}^{1, p}(Z)} \varphi_{0,+} & <0=\varphi_{0,+}(0) .
\end{aligned}
$$

By hypothesis we have $j(z, x)=\int_{0}^{x} f(z, r) d r$ for almost all $z \in Z$ and all $x \in \mathbb{R}$. So using hypothesis $\mathrm{H}(j)_{3}$ (iii), it follows that

$$
\left|\widehat{j}_{+}(z, x)\right| \leq \widehat{\alpha}(z) \quad \text { for a.a. } z \in Z \text {, all } x \in \mathbb{R} \text {, with } \widehat{\alpha} \in L^{\infty}(Z)_{+} .
$$

Therefore from this and Poincare's inequality, we infer that $\varphi_{0,+}$ is coercive. It is easy to see that $\varphi_{0,+}$ is weakly lower semicontinuous on $W_{0}^{1, p}(Z)$. So by the Weierstrass theorem, we can find $\widehat{y}_{0}$ a minimizer of $\varphi_{0,+}$ and $\varphi_{0,+}\left(\widehat{y}_{0}\right)<0=\varphi_{0,+}(0)$, that is, $\widehat{y}_{0} \neq 0$ (see (4.66)). Since $\widehat{y}_{0}$ is a nonzero critical point of $\varphi_{0,+}$, we must have $\widehat{y}_{0}=y_{+}$. Clearly $y_{+}$is a local $C_{0}^{1}(\bar{Z})$-minimizer of $\varphi_{0}$ and so $y_{+}$is a local $W_{0}^{1, p}(Z)$ minimizer of $\varphi_{0}$. We can assume that $y_{+}$is an isolated critical point of $\varphi_{0}$. If this is not the case, we can find a sequence $\left\{x_{n}\right\}_{n \geq 1} \subseteq W_{0}^{1, p}(Z)$ of critical points of $\varphi_{0}$ such that

$$
x_{n} \longrightarrow y_{+} \text {in } W_{0}^{1, p}(Z) \quad \text { as } n \longrightarrow \infty, \quad x_{n} \neq 0, y_{+}, y_{-} \quad \forall n \geq 1
$$

Since $x_{n}$ is a critical point of $\varphi_{0}$, we must have $x_{n} \in I$. Thus we have produced a whole sequence of distinct nontrivial nodal solutions for problem (1.1).

Similarly working with $\varphi_{0_{-}}$on $I_{-}$, we have that $y_{-}$is a global minimizer of $\varphi_{0,-}, \varphi_{0,-}\left(y_{-}\right)=\varphi_{0}\left(y_{-}\right)<0=\varphi_{0}(0)$ and we can assume that it is an isolated critical point of $\varphi_{0}$. As in Motreanu et al. [36], we can find $\delta>0$ small such that

$$
\begin{aligned}
& \varphi_{0}\left(y_{-}\right)<\inf \left[\varphi_{0}(x): x \in \partial B_{\delta}\left(y_{-}\right)\right] \leq 0 \\
& \varphi_{0}\left(y_{+}\right)<\inf \left[\varphi_{0}(x): x \in \partial B_{\delta}\left(y_{+}\right)\right] \leq 0
\end{aligned}
$$

where $\partial B_{\delta}\left(y_{ \pm}\right)=\left\{x \in W_{0}^{1, p}(Z):\left\|x_{0}-y_{ \pm}\right\|=\delta\right\}$.

If we set $S=\partial B_{\delta}\left(y_{+}\right) \cup \partial B_{\delta}\left(y_{-}\right), I=\left[y_{-}, y_{+}\right]$, and $I_{0}=\left\{y_{-}, y_{+}\right\}$, then we can easily see that the pair $\left\{I_{0}, I\right\}$ is linking with $S$ in $W_{0}^{1, p}(Z)$. Moreover, as for $\varphi_{0,+}$ we can check that $\varphi_{0}$ is coercive and so we can easily verify the PS-condition. Therefore, we can apply Theorem 2.2 and produce $y_{0} \in W_{0}^{1, p}(Z)$, a critical point of $\varphi_{0}$, such that

$$
\varphi_{0}\left(y_{ \pm}\right)<\varphi_{0}\left(y_{0}\right)=\inf _{\bar{\gamma} \in \bar{\Gamma}} \max _{t \in[-1,1]} \varphi_{0}(\bar{\gamma}(t))
$$


where $\bar{\Gamma}=\left\{\bar{\gamma} \in C([-1,1]): \bar{\gamma}(-1)=y_{-}, \bar{\gamma}(1)=y_{+}\right\}$. Note that from (4.70) we have that $y_{0} \neq y_{ \pm}$.

We claim that $\varphi_{0}\left(y_{0}\right)<0=\varphi_{0}(0)$ and so $y_{0} \neq 0$. To show this, it is enough to produce a path $\bar{\gamma}_{0} \in \bar{\Gamma}$ such that

$$
\varphi_{0}\left(\bar{\gamma}_{0}(t)\right)<0 \quad \forall t \in[-1,1]
$$

So in what follows we construct such a path $\bar{\gamma}_{0}$.

Recall that $\partial B_{1}^{L^{p}(Z)}=\left\{x \in L^{p}(Z):\|x\|_{p}=1\right\}$ and $S=W_{0}^{1, p}(Z) \cap \partial B_{1}^{L^{p}(Z)}$ endowed with the $W_{0}^{1, p}(Z)$-topology. We also set

$$
S_{c}=W_{0}^{1, p}(Z) \cap C_{0}^{1}(\bar{Z}) \cap \partial B_{1}^{L^{p}(Z)}
$$

equipped with the $C_{0}^{1}(\bar{Z})$-topology.

Then $S_{c}$ is dense in $S$ in the $W_{0}^{1, p}(Z)$-topology. Because of (2.10), given $\delta>0$, we can find $\widehat{\gamma}_{0} \in \bar{\Gamma}_{0}=\left\{\gamma_{0} \in C([-1,1], S): \gamma_{0}(-1)=-u_{1}, \gamma_{0}(1)=u_{1}\right\}$ satisfying $\widehat{\gamma}_{0}([-1,1]) \subseteq S_{c}$ and

$$
\max \left[\|D x\|_{p}^{p}: x \in \widehat{\gamma}_{0}([-1,1])\right] \leq \lambda_{2}+\delta
$$

(since $C\left([-1,1], S_{c}\right)$ is dense in $C([-1,1], S)$.

We can always choose $\delta>0$ small such that

$$
\lambda_{2}+2 \delta<\liminf _{x \rightarrow 0} \frac{u}{|x|^{p-2} x} \text { uniformly for a.a. } z \in Z, \text { all } u \in \partial j(z, x)
$$

(see hypothesis $\left.H(j)_{3}(\mathrm{v})\right)$. Then we can find $\delta_{0}>0$ such that

$$
\lambda_{2}+\delta<\frac{u}{|x|^{p-2} x} \quad \text { for a.a. } z \in Z \text {, all } 0<|x| \leq \delta_{0} \text {, and all } u \in \partial j(z, x) .
$$

As before exploiting the fact that $(d / d x) f(z, x) \in \partial j(z, x)$ for a.a. $z \in Z$ and almost all $x \in \mathbb{R}$, from (4.75) we obtain

$$
\frac{1}{p}\left(\lambda_{2}+\delta\right)|x|^{p}<j(z, x) \quad \text { for a.a. } z \in Z \text { and all } 0<|x| \leq \delta_{0}
$$

Since $\hat{\gamma}_{0}([-1,1]) \subseteq S_{c}$ and $-y_{-}, y_{+} \in \operatorname{int} C_{0}^{1}(\bar{Z})_{+}$, we can find $\varepsilon>0$ small such that

$$
\begin{gathered}
|\varepsilon x(z)| \leq \delta_{0} \quad \forall z \in \bar{Z}, \text { all } x \in \widehat{\gamma}_{0}([-1,1]), \\
\varepsilon x \in\left[-y_{-}, y_{+}\right] \text {and all } x \in \widehat{\gamma}_{0}([-1,1]) .
\end{gathered}
$$


If $x \in \widehat{\gamma}_{0}([-1,1])$, we have

$$
\begin{aligned}
\varphi_{0}(\varepsilon x) & =\varphi(\varepsilon x)=\frac{\varepsilon^{p}}{p}\|D x\|_{p}^{p}-\int_{Z} j(z, \varepsilon x(z)) d z \\
& <\frac{\varepsilon^{p}}{p}\|D x\|_{p}^{p}-\frac{\varepsilon^{p}}{p}\left(\lambda_{2}+\delta\right)\|x\|_{p}^{p} \quad(\text { see }(4.76)) \\
& \leq 0 \quad\left(\text { see }(4.73) \text { and recall }\|x\|_{p}=1\right) .
\end{aligned}
$$

So, if we consider the continuous path $\gamma_{0}=\varepsilon \widehat{\gamma}_{0}$ which joins $-\varepsilon u_{1}$ and $\varepsilon u_{1}$, we have

$$
\left.\varphi_{0}\right|_{\gamma_{0}}<0
$$

Next with the help of Theorem 2.5, we will produce a continuous path joining $\varepsilon u_{1}$ and $y_{+}$along which $\widehat{\varphi}$ is strictly negative. We know that $\left\{0, y_{+}\right\}$are the only critical points of $\varphi_{0,+}$. Let $\alpha_{+}=\varphi_{0,+}\left(y_{+}\right)=\inf \varphi_{0,+}<0$ and let $b_{+}=0$. Recall that $\varphi_{0,+}$ is coercive and so it satisfies the PS-condition. Therefore according to Theorem 2.5, we can find a deformation $h:[0,1] \times{\stackrel{0}{\varphi_{0}}}^{b_{+}} \rightarrow{\stackrel{0}{\varphi_{0}}}^{b_{+}}$such that

$$
\begin{aligned}
\left.h(t, \cdot)\right|_{K_{\alpha_{+}}} & =i d \quad \forall t \in[0,1], \\
h\left(1, \varphi_{0,+}^{0} b_{+}\right. & \subseteq \varphi_{0,+}^{0} \quad \cup K_{a_{+}} \\
\varphi_{0,+}(h(t, z)) & \subseteq \varphi_{0,+}(x) \quad \forall(t, x) \in[0,1] \times \varphi_{0,+}^{0} b^{b_{+}} .
\end{aligned}
$$

We consider the path $\gamma_{+}:[0,1] \rightarrow \varphi_{0,+}^{b^{b_{+}}}$defined by

$$
\gamma_{+}(t)=h\left(t, \varepsilon u_{1}\right) \quad \forall t \in[0,1]
$$

Clearly this is a continuous path and we have

$$
\begin{gathered}
\gamma_{+}(0)=h\left(0, \varepsilon u_{1}\right)=\varepsilon u_{1} \quad(\text { since } h \text { is a deformation), } \\
\gamma_{+}(1)=h\left(1, \varepsilon u_{1}\right)=y_{+}\left(\varphi_{0,+}^{0} a_{+}=\varnothing, K_{a_{+}}=\left\{y_{+}\right\}\right), \\
\left.\varphi_{0,+}\left(\gamma_{+}(t)\right)=\varphi_{0,+}\left(h\left(t, \varepsilon u_{1}\right)\right) \leq \varphi_{0,+}\left(\varepsilon u_{1}\right)<0 \quad \forall t \in[0,1] \text { (see }(4.79)\right) .
\end{gathered}
$$

Therefore we have produced a continuous path $\gamma_{+}$joining $\varepsilon u_{1}$ and $y_{+}$such that

$$
\left.\varphi_{0,+}\right|_{\gamma_{+}}<0
$$

But note that $\varphi_{0,+} \geq \varphi_{0}\left(\right.$ see $\left.H(j)_{3}(v i)\right)$. Hence

$$
\left.\varphi_{0}\right|_{\gamma_{+}}<0
$$


In a similar fashion, we produce a continuous path $\gamma_{\text {- joining }}-\varepsilon u_{1}$ and $y_{-}$such that

$$
\left.\varphi_{0}\right|_{\gamma_{-}}<0
$$

If we join the paths $\gamma_{-}, \gamma_{0}, \gamma_{+}$, we produce a continuous path $\bar{\gamma}_{0} \in \bar{\Gamma}$ such that

$$
\left.\varphi_{0}\right|_{\bar{\gamma}_{0}}<0, \quad(\text { see }(4.79),(4.84),(4.85)) .
$$

From (4.70) it follows that $\varphi_{0}\left(y_{0}\right)<0=\widehat{\varphi}(0)$ and so $y_{0} \neq 0$.

Therefore $y_{0}$ is the third nontrivial solution of (1.1), which is (nodal) and from the nonlinear regularity theory we have $y_{0} \in C_{0}^{1}(\bar{Z})$.

\section{Acknowledgments}

The authors wish to thank the referees for their remarks. Researcher supported by a grant of the National Scholarship Foundation of Greece (I.K.Y.).

\section{References}

[1] F. H. Clarke, Optimization and Nonsmooth Analysis, Canadian Mathematical Society Series of Monographs and Advanced Texts, John Wiley \& Sons, New York, NY, USA, 1983.

[2] Z. Naniewicz and P. D. Panagiotopoulos, Mathematical Theory of Hemivariational Inequalities and Applications, vol. 188 of Monographs and Textbooks in Pure and Applied Mathematics, Marcel Dekker, New York, NY, USA, 1995.

[3] A. Ambrosetti, J. Garcia Azorero, and I. Peral Alonso, "Multiplicity results for some nonlinear elliptic equations," Journal of Functional Analysis, vol. 137, no. 1, pp. 219-242, 1996.

[4] J. García Azorero and I. Peral Alonso, "Some results about the existence of a second positive solution in a quasilinear critical problem," Indiana University Mathematics Journal, vol. 43, no. 3, pp. 941-957, 1994.

[5] J. P. García Azorero, I. Peral Alonso, and J. J. Manfredi, "Sobolev versus Hölder local minimizers and global multiplicity for some quasilinear elliptic equations," Communications in Contemporary Mathematics, vol. 2, no. 3, pp. 385-404, 2000.

[6] E. N. Dancer and Y. Du, "On sign-changing solutions of certain semilinear elliptic problems," Applicable Analysis, vol. 56, no. 3-4, pp. 193-206, 1995.

[7] Z. Zhang and X. Li, "Sign-changing solutions and multiple solutions theorems for semilinear elliptic boundary value problems with a reaction term nonzero at zero," Journal of Differential Equations, vol. 178, no. 2, pp. 298-313, 2002.

[8] S. Carl and K. Perera, "Sign-changing and multiple solutions for the $p$-Laplacian," Abstract and Applied Analysis, vol. 7, no. 12, pp. 613-625, 2002.

[9] Z. Zhang and S. Li, “On sign-changing and multiple solutions of the $p$-Laplacian," Journal of Functional Analysis, vol. 197, no. 2, pp. 447-468, 2003.

[10] Z. Zhang, J. Chen, and S. Li, "Construction of pseudo-gradient vector field and sign-changing multiple solutions involving p-Laplacian," Journal of Differential Equations, vol. 201, no. 2, pp. 287303, 2004.

[11] M. E. Filippakis and N. S. Papageorgiou, "Multiple constant sign and nodal solutions for nonlinear elliptic equations with the p-Laplacian," Journal of Differential Equations, vol. 245, no. 7, pp. 1883-1922, 2008.

[12] Z. Zhang and K. Perera, "Sign changing solutions of Kirchhoff type problems via invariant sets of descent flow," Journal of Mathematical Analysis and Applications, vol. 317, no. 2, pp. 456-463, 2006.

[13] B. Ahmad and J. J. Nieto, "The monotone iterative technique for three-point second-order integrodifferential boundary value problems with p-Laplacian," Boundary Value Problems, vol. 2007, Article ID 57481, 9 pages, 2007. 
[14] C.-G. Kim, Y.-H. Lee, and I. Sim, "Multiplicity results of positive radial solutions for $p$-Laplacian problems in exterior domains," Boundary Value Problems, vol. 2008, Article ID 395080, 18 pages, 2008.

[15] X. Lin, W. Sun, and D. Jiang, "Existence and uniqueness of solutions for boundary value problems to the singular one-dimension p-Laplacian," Boundary Value Problems, vol. 2008, Article ID 194234, 8 pages, 2008.

[16] M. Väth, "Continuity, compactness, and degree theory for operators in systems involving $p$ Laplacians and inclusions," Journal of Differential Equations, vol. 245, no. 5, pp. 1137-1166, 2008.

[17] J.-N. Corvellec, "On the second deformation lemma," Topological Methods in Nonlinear Analysis, vol. 17, no. 1, pp. 55-66, 2001.

[18] D. Averna, S. A. Marano, and D. Motreanu, "Multiple solutions for a Dirichlet problem with $p$ Laplacian and set-valued nonlinearity," Bulletin of the Australian Mathematical Society, vol. 77, no. 2, pp. 285-303, 2008.

[19] L. Gasiński and N. S. Papageorgiou, Nonsmooth Critical Point Theory and Nonlinear Boundary Value Problems, vol. 8 of Series in Mathematical Analysis and Applications, Chapman \& Hall/CRC, Boca Raton, Fla, USA, 2005.

[20] D. Motreanu and P. D. Panagiotopoulos, Minimax Theorems and Qualitative Properties of the Solutions of Hemivariational Inequalities, vol. 29 of Nonconvex Optimization and Its Applications, Kluwer Academic Publishers, Dordrecht, The Netherlands, 1999.

[21] L. Gasiński and N. S. Papageorgiou, Nonlinear Analysis, vol. 9 of Series in Mathematical Analysis and Applications, Chapman \& Hall/CRC, Boca Raton, Fla, USA, 2006.

[22] F. Cuccu, B. Emamizadeh, and G. Porru, "Optimization on the first eigenvalue in problems involving the $p$-Laplacian," Proceedings of the American Mathematical Society, vol. 137, pp. 1677-1687, 2009.

[23] J. L. Vázquez, "A strong maximum principle for some quasilinear elliptic equations," Applied Mathematics and Optimization, vol. 12, no. 3, pp. 191-202, 1984.

[24] A. Anane and N. Tsouli, “On the second eigenvalue of the $p$-Laplacian," in Nonlinear Partial Differential Equations, A. Benikrane and J. P. Gossez, Eds., vol. 343 of Pitman Research Notes in Mathematics Series, pp. 1-9, Longman, Harlow, UK, 1996.

[25] M. Cuesta, D. de Figueiredo, and J.-P. Gossez, "The beginning of the Fučik spectrum for the $p$ Laplacian," Journal of Differential Equations, vol. 159, no. 1, pp. 212-238, 1999.

[26] H. Amann and E. Zehnder, "Nontrivial solutions for a class of nonresonance problems and applications to nonlinear differential equations," Annali della Scuola Normale Superiore di Pisa. Classe di Scienze. Serie IV, vol. 7, no. 4, pp. 539-603, 1980.

[27] K.-C. Chang, "Variational methods for nondifferentiable functionals and their applications to partial differential equations," Journal of Mathematical Analysis and Applications, vol. 80, no. 1, pp. 102-129, 1981.

[28] M. Guedda and L. Véron, "Quasilinear elliptic equations involving critical Sobolev exponents," Nonlinear Analysis: Theory, Methods E Applications, vol. 13, no. 8, pp. 879-902, 1989.

[29] D. Motreanu and N. S. Papageorgiou, "Multiple solutions for nonlinear elliptic equations at resonance with a nonsmooth potential," Nonlinear Analysis: Theory, Methods \& Applications, vol. 56, no. 8, pp. 1211-1234, 2004.

[30] Z. Jin, "Multiple solutions for a class of semilinear elliptic equations," Proceedings of the American Mathematical Society, vol. 125, no. 12, pp. 3659-3667, 1997.

[31] S. Li and Z.-Q. Wang, "Mountain pass theorem in order intervals and multiple solutions for semilinear elliptic Dirichlet problems," Journal d'Analyse Mathématique, vol. 81, no. 1, pp. 373-396, 2000.

[32] M. Marcus and V. J. Mizel, "Absolute continuity on tracks and mappings of Sobolev spaces," Archive for Rational Mechanics and Analysis, vol. 45, no. 4, pp. 294-320, 1972.

[33] S. Carl and S. Heikkilä, Nonlinear Differential Equations in Ordered Spaces, vol. 111 of Monographs and Surveys in Pure and Applied Mathematics, Chapman \& Hall/CRC, Boca Raton, Fla, USA, 2000.

[34] N. Dunford and J. Schwartz, Linear Operators. I. General Theory, John Wiley \& Sons, Wiley-Interscience, 1958.

[35] S. Hu and N. S. Papageorgiou, Handbook of Multivalued Analysis. Volume I: Theory, vol. 419 of Mathematics and Its Applications, Kluwer Academic Publishers, Dordrecht, The Netherlands, 1997.

[36] D. Motreanu, V. V. Motreanu, and N. S. Papageorgiou, "A degree theoretic approach for multiple solutions of constant sign for nonlinear elliptic equations," Manuscripta Mathematica, vol. 124, no. 4, pp. 507-531, 2007. 